TUM-HEP-779/10

\title{
Footprints of SUSY GUTs in Flavour Physics
}

\author{
Andrzej J. Buras, ${ }^{1,2}$ Minoru Nagai, ${ }^{3}$ and Paride Paradisi ${ }^{1}$ \\ ${ }^{1}$ Physik-Department, Technische Universität München, \\ James-Franck-Straße, D-85748 Garching, Germany \\ ${ }^{2}$ TUM Institute for Advanced Study, Technische Universität München, \\ Lichtenbergstr. 2a, D-85748 Garching, Germany \\ ${ }^{3}$ Excellence Cluster Universe, Technische Universität München, D-85748 Garching, Germany
}

\begin{abstract}
Supersymmetric (SUSY) Grand Unified theories (GUTs) generally predict FCNC and CP violating processes to occur both in the leptonic and hadronic sectors. Assuming an underlying $S U(5)$ group plus right-handed neutrinos $(\mathrm{RN})$, we perform an extensive study of FCNC and CP violation, analyzing the correlations between leptonic and hadronic processes like $\mu \rightarrow e \gamma$ and $K^{0}-\bar{K}^{0}$ mixing, $\tau \rightarrow \mu \gamma$ and $b \rightarrow s$ transitions such as $B_{d} \rightarrow \phi K_{s}$ and $B_{s}^{0}-\bar{B}_{s}^{0}$ mixing. Moreover, we examine the impact of the considered scenario on the UT analyses, monitoring the low energy consequences implied by possible solutions to the various tensions in the present UT analyses. We compare the phenomenological implications of this NP scenario with the ones of supersymmetric flavour models finding a few striking differences that could allow to distinguish these different NP models.
\end{abstract}

\section{INTRODUCTION}

Grand unified theories (GUTs) 1 predict a very attractive unification of the strong and electroweak interactions and, when embedded in a supersymmetric framework, they also lead to a very successful gauge coupling unification [2].

Since a direct access to the GUT scale $\left(M_{G}\right)$ is not possible, experimental signals for GUTs may be looked for either by confronting their predictions for quantities that are not predicted in the SM, such as the weak mixing angle and the $m_{b} / m_{\tau}$ ratio or by the observation of processes which are forbidden or highly suppressed in the $\mathrm{SM}$, such as the proton decay. In particular, the baryon number $B$, the family lepton numbers $L_{e}, L_{\mu}, L_{\tau}$ and the total lepton number $L=L_{e}+L_{\mu}+L_{\tau}$, which are accidental symmetries of the SM, are broken in a GUT context. As a result, processes violating $B, L$ or $L_{i}$ are generated and they turn out to be suppressed by powers of $1 / M_{G}$. Still, the experimental sensitivity to proton decay and to neutrino masses (assuming an underlying see-saw mechanism [3]) allow to probe $M_{G}$. Indeed, the observed neutrino masses and mixings give solid indications for the existence of some New Physics (NP) at $M_{G}$. In contrast, $L_{i}$ violating processes, such as $\mu \rightarrow e \gamma$, are predicted to be far below any realistic experimental sensitivity.

If the theory above $M_{G}$ is supersymmetric, the situation drastically changes. The dynamics at the scale $M_{G}$ leave indelible traces on the soft terms of the light sparticles by means of interactions not suppressed by inverse powers of $M_{G}$ [4, 5]. Processes which violate $L_{i}$ are now suppressed only by powers of $1 / \tilde{m}$, where $\tilde{m}$ is the scale of supersymmetry breaking, and therefore they might be measurable.

Moreover, since within GUTs quarks and leptons sit in the same multiplets, the quark-lepton unification feeds into the SUSY breaking soft sector [4, 6, 7].

This implies relations between lepton and quark flavor changing transitions at the weak scale. For example, one can expect correlations between $\mu \rightarrow e \gamma$ and $K^{0}-\bar{K}^{0}$ mixing, $\tau \rightarrow \mu \gamma$ and $b \rightarrow s$ transitions such as $B_{d} \rightarrow \phi K_{s}$ and $B_{s}^{0}-\bar{B}_{s}^{0}$ mixing and so on. Therefore, hadronic and leptonic FCNC processes provide a splendid opportunity to link the weak scale to the GUT scale, where the fundamental SUSY Lagrangian is defined.

Despite of the remarkable agreement of flavour data with the SM predictions in the $K$ and $B_{d}$ systems, a closer look at the data might indicate some tensions especially in $\mathrm{CP}$ violating observables. In particular:

i) the most recent UT analyses 811 show that the size of $\mathrm{CP}$ violation determined through the $B_{d}^{0}-\bar{B}_{d}^{0}$ system, appears insufficient to describe the experimental value of $\epsilon_{K}$ within the SM if the $\Delta M_{d} / \Delta M_{s}$ constraint is taken into account 9. Vice versa, the simultaneous SM description of $\epsilon_{K}$ and $\Delta M_{d} / \Delta M_{s}$ requires a $\sin 2 \beta$ significantly larger than the measured value of $S_{\psi K_{S}}[8]$.

ii) The recent messages from the Tevatron seem to hint the presence of new sources of CPV entering the $B_{s}^{0}$ system [12 14].

iii) The value of $\sin 2 \beta$ extracted from some penguin dominated modes, such as $B_{d} \rightarrow \phi K_{S}$, is significantly lower than the value from $B_{d} \rightarrow \psi K_{S}$.

iv) Last but not least, we remind the $(g-2)_{\mu}$ anomaly [15. Interestingly, the possibility that the present $3 \sigma$ discrepancy may arise from errors in the determination of the hadronic leading-order contribution to $\Delta a_{\mu}$ seems to be unlikely [16].

In the light of the above considerations, in this work we focus on the SUSY $S U(5)$ GUT model plus right-handed neutrinos [17 $\left(S S U(5)_{R N}\right)$, accounting for the neutrino masses and mixings via a type-I sees-saw model [3], with the main goals:

i) to analyze how the $S S U(5)_{R N}$ model faces the above tensions, monitoring the low energy consequences implied by their possible solutions;

ii) to quantify the NP room left for $b \rightarrow s$ transitions compatible with all the available experimental data on $\Delta F=2$ and $\Delta F=1$ processes, 
iii) to outline strategies aimed to probe or to falsify the $S S U(5)_{R N}$ model by means of a correlated analysis of low energy observables, including also $K$ and $D$ systems as well as lepton flavour violation, electric dipole moments (EDMs) and the $(g-2)_{\mu}$.

Since many analyses along this subject appeared in the literature [18, we want to emphasize here that the current study goes well beyond previous works as for i) the inclusion of all relevant SUSY contributions, ii) the number of processes considered, and iii) the special attention given to the UT analyses. Moreover, we point out many new correlations among observables that should enable us to probe or falsify the scenario in question once improved data will be available.

In Section 2 we update our UT analysis of [19] using the $\left(R_{b}, \gamma\right)$ plane and stressing that a large value of $\gamma$ in the UT would provide a natural solution to the observed tensions within the $S S U(5)_{R N}$ model. In Section 3 we summarize the flavour structure of the SUSY GUT considered in this paper. In Sections 4 and 5 the relevant formulae for the hadronic and the leptonic sectors are given, respectively. Section 6 is devoted to approximate analytical expressions for various correlations between hadronic and leptonic observables that are then analyzed numerically in Section 7. In Section 8 we present a DNA-table for this GUT scenario and compare it with the ones of supersymmetric flavour models analyzed by us in [19. Finally we end our paper with a list of the most important findings.

\section{UT ANALYSIS}

In this section, we perform a unitarity triangle (UT) analysis in the framework of the $S S U(5)_{R N}$ model. We remind that there exist two different UTs: 1) the socalled reference unitarity triangle (RUT) 20], determined entirely from tree level decays hence, likely unaffected by any significant NP pollution, and 2) the universal unitarity triangle (UUT) [21] of models with constrained MFV, determined by means of loop-induced FCNC processes and hence potentially sensitive to NP effects. Therefore, a comparative UT analysis performed by means of the RUT and UUT may unveil NP effects. In particular, the above UTs are characterized by the following parameters

$$
\begin{array}{lll}
V_{u s} \equiv \lambda, V_{c b}, R_{b}, \gamma & \mathrm{RUT}, \\
V_{u s} \equiv \lambda, V_{c b}, R_{t}, \beta & \mathrm{UUT},
\end{array}
$$

where $R_{b} \equiv\left|V_{u d} V_{u b}^{*}\right| /\left|V_{c d} V_{c b}^{*}\right|, \quad R_{t} \equiv\left|V_{t d} V_{t b}^{*}\right| /\left|V_{c d} V_{c b}^{*}\right|$ and the angles $\beta$ and $\gamma$ are such that $V_{t d}=\left|V_{t d}\right| e^{-i \beta}$ and $V_{u b}=\left|V_{u b}\right| e^{-i \gamma}$. Moreover, the dictionary between $\left(R_{t}, \beta\right)$ and $\left(R_{b}, \gamma\right)$ reads

$$
\begin{aligned}
& R_{t}=\sqrt{1+R_{b}^{2}-2 R_{b} \cos \gamma}, \quad \cot \beta=\frac{1-R_{b} \cos \gamma}{R_{b} \sin \gamma}, \\
& R_{b}=\sqrt{1+R_{t}^{2}-2 R_{t} \cos \beta}, \quad \cot \gamma=\frac{1-R_{t} \cos \beta}{R_{t} \sin \beta} .
\end{aligned}
$$

In terms of physical observables we can write

$$
R_{t}=\frac{\xi}{\lambda} \sqrt{\frac{m_{B_{s}}}{m_{B_{d}}}} \sqrt{\frac{\Delta M_{d}}{\Delta M_{s}}} \sqrt{\frac{C_{B_{s}}}{C_{B_{d}}}}, \quad \sin \left(2 \beta+2 \varphi_{B_{d}}\right)=S_{\psi K_{S}}
$$

with the SM limit recovered for $C_{B_{q}}=1$ and $\varphi_{B_{d}}=0$.

The last observable that is relevant for our UT analysis is $\epsilon_{K}$. In the SM, $\epsilon_{K}$ can be written as [9]

$$
\begin{aligned}
\left|\epsilon_{K}\right| & =\kappa_{\epsilon} C_{\epsilon} \hat{B}_{K}\left|V_{c b}\right|^{2}\left|V_{u s}\right|^{2}\left(\frac{\left|V_{c b}\right|^{2}}{2} R_{t}^{2} \sin 2 \beta \eta_{t t} S_{0}\left(x_{t}\right)+\right. \\
& \left.+R_{t} \sin \beta\left(\eta_{c t} S_{0}\left(x_{c}, x_{t}\right)-\eta_{c c} x_{c}\right)\right)
\end{aligned}
$$

where $C_{\epsilon} \simeq 3.658 \times 10^{4}$ and all the parameters entering the above expression are reported in Table $\square$ As stressed in [9], the SM prediction for $\epsilon_{K}$ implied by the measured value of $S_{\psi K_{S}}=\sin 2 \beta$ may be too small to agree with experiment. The main reasons are the decreased value of $\hat{B}_{K}$ and the decreased value of $\epsilon_{K}$ in the SM arising from a multiplicative factor, estimated as $\kappa_{\epsilon}=0.94 \pm 0.02$ [22].

Taking into account also the recent calculation of the QCD factor $\eta_{c t}$ at the NNLO [23, that enhances the value of $\varepsilon_{K}$ by $3 \%$ [23], the total suppression of $\epsilon_{K} \propto$ $\hat{B}_{K} \kappa_{\epsilon}$ compared to the commonly used formulae is typically of order $15 \%$. Using the inputs of Table I. eq. (4) for the SM case (where $C_{B_{s}}=C_{B_{d}}=1$ ), and eq. (5), one finds 23.

$$
\left|\epsilon_{K}\right|^{\mathrm{SM}}=(1.90 \pm 0.26) \times 10^{-3},
$$

to be compared with the experimental measurement 27 .

$$
\left|\epsilon_{K}\right|^{\exp }=(2.229 \pm 0.010) \times 10^{-3} .
$$

In fig. 1. we show the above tensions in the $R_{b}-\gamma$ plane updating the analysis of [19] by the inclusion of the new values for $\kappa_{\epsilon}[22]$ and $\eta_{c t}[23]$.

In the upper left plot of fig. 1, we show the regions corresponding to the $1 \sigma$ allowed ranges for $\sin 2 \beta, R_{t}$ and $\left|\epsilon_{K}\right|^{\mathrm{SM}}$ as calculated by means of (4) and (5), respectively, using the numerical input parameters of tab. I. As shown, there are three different values of $\left(R_{b}, \gamma\right)$, dependently which two constraints are simultaneously applied.

Possible solutions to this tension can be obtained assuming:

1) a positive NP contribution to $\epsilon_{K}$, at the level of $\approx$ $+20 \%$, leaving $\sin 2 \beta$ and $\Delta M_{d} / \Delta M_{s}$ SM-like 9 .

2) $\epsilon_{K}$ and $\Delta M_{d} / \Delta M_{s}$ NP free while $S_{\psi K_{S}}$ affected by a NP phase in $B_{d}$ mixing with $\varphi_{B_{d}} \approx-5^{\circ}$ [8].

3) $\epsilon_{K}$ and $S_{\psi K_{S}}$ NP free while $\Delta M_{d} / \Delta M_{s}$ affected by NP at the level of $\approx-20 \%$ [19, requiring in turn an increased value of $R_{t}$ to fit the data.

Of course all these effects could be simultaneously at work. 


\begin{tabular}{|c|c|c|c|}
\hline parameter & value & parameter & value \\
\hline$F_{K}$ & $(155.8 \pm 1.7) \mathrm{MeV} 25,26$ & $m_{s}(2 \mathrm{GeV})$ & $0.105 \mathrm{GeV} 27$ \\
\hline$F_{B_{d}}$ & $(192.8 \pm 9.9) \mathrm{MeV}[25,26$ & $m_{d}(2 \mathrm{GeV})$ & $0.006 \mathrm{GeV}$ [27] \\
\hline$F_{B_{s}}$ & $(238.8 \pm 9.5) \mathrm{MeV}[25,26]$ & $\left|V_{t s}\right|$ & $0.040 \pm 0.00332$ \\
\hline$\hat{B}_{K}$ & $0.725 \pm 0.02625,26$ & $\left|V_{t b}\right|$ & $1 \pm 0.0632$ \\
\hline$\hat{B}_{B_{d}}$ & $1.26 \pm 0.11,25,26$ & $\left|V_{t d}\right|_{\text {tree }}$ & $(8.3 \pm 0.5) \cdot 10^{-3} 32$ \\
\hline$\hat{B}_{B_{s}}$ & $1.33 \pm 0.0625,26$ & $\left|V_{u s}\right|$ & $0.2255 \pm 0.001927$ \\
\hline$M_{B_{s}}$ & $5.3664 \mathrm{GeV}[27]$ & $\left|V_{c b}\right|$ & $(40.6 \pm 1.1) \times 10^{-3} 33$ \\
\hline$M_{B_{d}}$ & $5.2795 \mathrm{GeV} 27$ & $\sin (2 \beta)_{\text {tree }}$ & $0.734 \pm 0.03832$ \\
\hline$M_{K}$ & $0.497614 \mathrm{GeV}$ [27] & $\sin \left(2 \beta_{s}\right)$ & $0.038 \pm 0.00332$ \\
\hline$\eta_{c c}$ & $1.43 \pm 0.2328$ & $\alpha_{s}\left(m_{Z}\right)$ & 0.118434 \\
\hline$\eta_{t t}$ & $0.5765 \pm 0.006529$ & $\Delta M_{s}$ & $(17.77 \pm 0.12) \mathrm{ps}^{-1}$ \\
\hline$\eta_{c t}$ & $0.496 \pm 0.04723$ & $\Delta M_{d}$ & $(0.507 \pm 0.005) \mathrm{ps}^{-1}$ \\
\hline$\eta_{B}$ & $0.551 \pm 0.00729$ & $\Delta M_{K}$ & $(5.292 \pm 0.009) \cdot 10^{-3} \mathrm{ps}^{-1} 27$ \\
\hline & $1.243 \pm 0.02825,26$ & & $0.94 \pm 0.02[22]$ \\
\hline$m_{c}\left(m_{c}\right)$ & $(1.268 \pm 0.009) \mathrm{GeV}[30]$ & $\varepsilon_{K}^{\exp }$ & $(2.229 \pm 0.01) \cdot 10^{-3} 27$ \\
\hline$m_{t}\left(m_{t}\right)$ & $(163.7 \pm 1.1) \mathrm{GeV} 31$ & $S_{\psi K_{S}}^{\exp }$ & $0.672 \pm 0.023$ \\
\hline$m_{b}\left(m_{b}\right)$ & $(4.2+0.17-0.07) \mathrm{GeV}[27]$ & & \\
\hline
\end{tabular}

TABLE I: Values of the input parameters used in our analysis. The subscript "tree" in $\left|V_{t d}\right|$ and $\sin (2 \beta)$ stands for the inputs extracted from data using only tree-level observables [32].

As stressed in 19, the possibility 3) implies a large value of $\gamma$, as shown in fig. 1 (see the lower plot on the right), and $\alpha$ significantly below $90^{\circ}$. Therefore, this scenario can be easily probed or falsified by means of improved determinations of $\gamma$, as expected at the $\mathrm{LHCb}$, and $\alpha$. Moreover, the possibility 3 ) is particularly relevant within the $S S U(5)_{R N}$ model, as we will discuss in detail later. In such a case, if the mixing angle regulating the $b \rightarrow s$ transition contains a natural $\mathcal{O}(1) \mathrm{CPV}$ phase, then solution 3) also implies a non-standard value for $S_{\psi \phi}$ in the $B_{s}^{0}$ system.

\section{SUSY GUTS AND FLAVOUR PHENOMENOLOGY}

The quark-lepton unification predicted by GUTs implies, in a SUSY framework, a unification of the squark and slepton mass matrices at the GUT scale.

For instance, within $\mathrm{SU}(5)$, the multiplet $\overline{\mathbf{5}}$ contains the right-handed down-type quarks $\left(D^{c}\right)$ and the left-handed lepton doublets $(L)$ while the $\mathbf{1 0}$ multiplet contains the left-handed quark doublets $(Q)$, the right-handed up-type quarks $\left(U^{c}\right)$, and the right-handed charged-leptons $\left(E^{c}\right)$.

Even if we assume a complete flavour blindness for the sfermion masses at the high scale (either $M_{P}$ or $M_{G}$ ), we can still expect sizable sources of FCNC if the theory contains neutrino Yukawa interactions accounting for the neutrino masses and mixings by means of a see-saw mechanism.

Assuming a type-I see-saw with three heavy right- handed neutrinos 3, the effective light-neutrino mass matrix resulting after integrating out the heavy fields is $m_{\nu}=y_{\nu}^{T} \hat{M}_{\nu}^{-1} y_{\nu}\left\langle H_{u}\right\rangle^{2}$ where $\hat{M}_{\nu}$ is the right-handed neutrino mass matrix, $y_{\nu}$ is the unknown neutrino Yukawa matrix, and $\left\langle H_{u}\right\rangle$ is the up-type Higgs VEV. Hereafter, we take a basis where $\hat{M}_{\nu}$ is diagonal and symbols with hat mean they are diagonal matrices.

In the mSUGRA scenario and specializing to the case of $S S U(5)_{R N}$ model, low-energy flavor-violating SUSYbreaking terms are radiatively induced, and they are qualitatively given as

$$
\begin{aligned}
& \left(m_{\tilde{d}_{R}}^{2}\right)_{i j}=-\frac{\left(3 m_{0}^{2}+A_{0}^{2}\right)}{8 \pi^{2}}\left(e^{i \hat{\phi}_{d}} y_{\nu}^{T} y_{\nu}^{*} e^{-i \hat{\phi}_{d}}\right)_{i j} \ln \frac{M_{\mathrm{P}}}{M_{\mathrm{G}}}, \\
& \left(m_{\tilde{d}_{L}}^{2}\right)_{i j}=-\frac{\left(3 m_{0}^{2}+A_{0}^{2}\right)}{8 \pi^{2}}\left(V^{\dagger} \hat{y}_{u}^{2} V\right)_{i j}\left(3 \ln \frac{M_{\mathrm{P}}}{M_{\mathrm{G}}}+\ln \frac{M_{\mathrm{G}}}{\tilde{m}}\right), \\
& \left(m_{\tilde{e}_{R}}^{2}\right)_{i j}=-3 \frac{\left(3 m_{0}^{2}+A_{0}^{2}\right)}{8 \pi^{2}}\left(e^{i \hat{\phi}_{d}} V^{T} \hat{y}_{u}^{2} V^{*} e^{-i \hat{\phi}_{d}}\right)_{i j} \ln \frac{M_{\mathrm{P}}}{M_{\mathrm{G}}}, \\
& \left(m_{\tilde{l}_{L}}\right)_{i j}=-\frac{\left(3 m_{0}^{2}+A_{0}^{2}\right)}{8 \pi^{2}}\left(y_{\nu}^{\dagger}\right)_{i k}\left(y_{\nu}\right)_{k j} \ln \frac{M_{\mathrm{P}}}{M_{\nu_{k}}},
\end{aligned}
$$

where $V$ is the CKM matrix, $\hat{\phi}_{d}$ is a GUT phase and $m_{0}$ $\left(A_{0}\right)$ is the universal scalar mass (trilinear coupling).

Within $S U(5)$, as both $Q$ and $E^{c}$ are hosted in the 10 representation, the CKM matrix mixing of the lefthanded quarks will give rise to off-diagonal entries in the running of the right-handed slepton soft masses $\left(m_{\tilde{e}_{R}}^{2}\right)_{i j}$ due to the interaction of the colored Higgs [4, 6]. Vice versa, $Y_{\nu}$ enters the mass matrices of both right-handed down squark and left-handed sleptons, as $D^{c}$ and $L$ lie in the $\overline{\mathbf{5}}$ multiplet of $S U(5)$. 

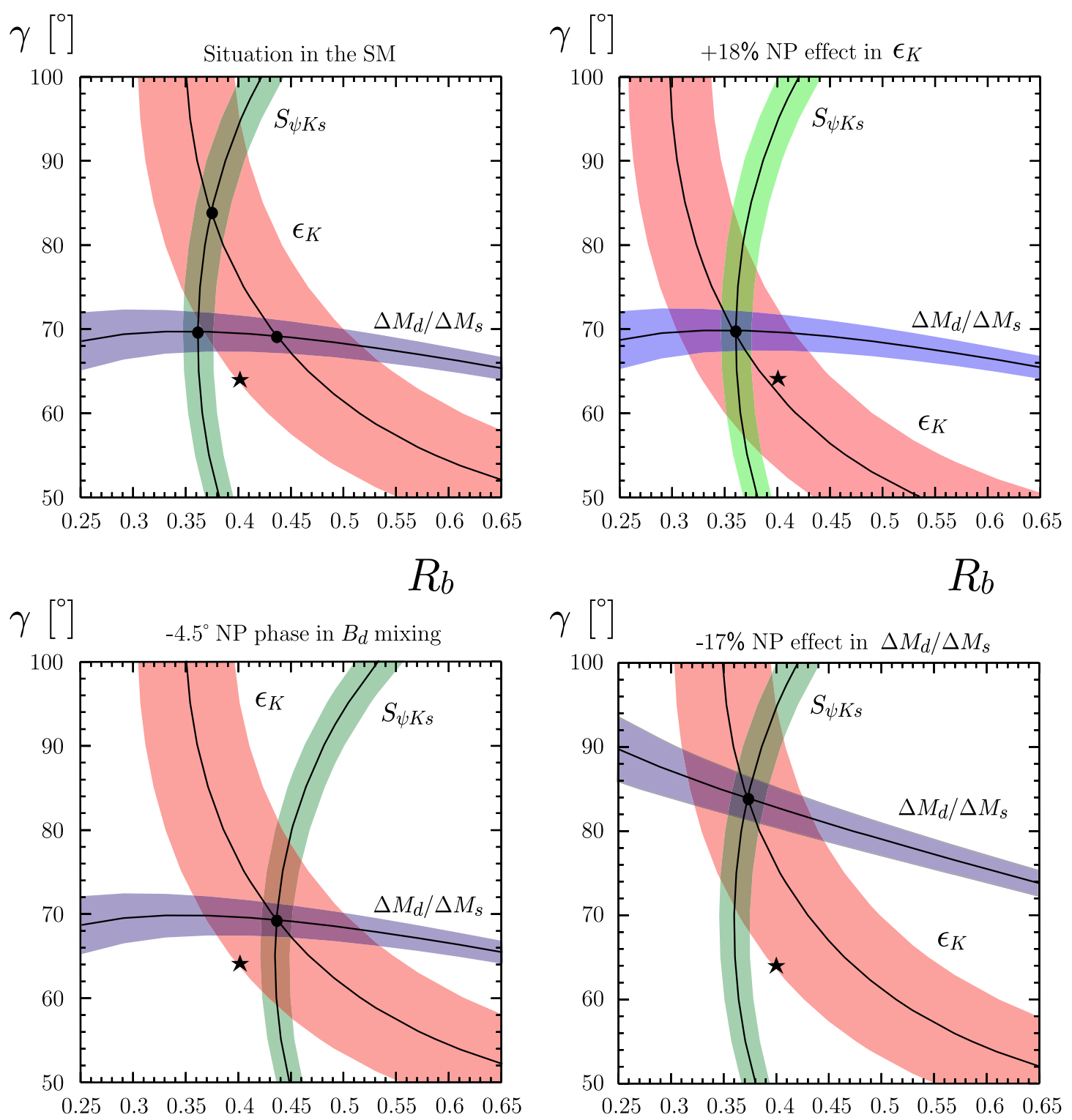

$R b$

$R b$

FIG. 1: The $R_{b}-\gamma$ plane assuming: i) the SM situation with the input parameters of Table $\mathrm{I}$ (upper left), ii) sin $2 \beta$ and $R_{t}$ NP free while $\epsilon_{K}$ affected by a $+18 \%$ NP effect compared to the SM contribution (upper right), iv) $\epsilon_{K}$ and $R_{t}$ NP free while $\sin 2 \beta$ affected by a NP phase in $B_{d}$ mixing with $\varphi_{B_{d}} \approx-4.5^{\circ}$ (lower left), iii) $\epsilon_{K}$ and $\sin 2 \beta$ NP free while $\Delta M_{d} / \Delta M_{s}$ affected by a $-20 \%$ NP contribution compared to the SM contribution (lower right). The black star stands for the values obtained from the NP UT fit [24].

We remind that, within a type-I see-saw scenario, $y_{\nu}$ can be written in the general form [36] $y_{\nu}=$ $\sqrt{\hat{M}_{\nu}} R \sqrt{\hat{m}_{\nu}} U^{\dagger} /\left\langle H_{u}\right\rangle$ where $R$ is an arbitrary complex orthogonal matrix while $U$ is the PMNS matrix. The determination of $\left(m_{\tilde{l}_{L}}^{2}\right)_{i \neq j}$ would require a complete knowledge of the neutrino Yukawa matrix $y_{\nu}$, which is not possible using only low-energy observables from the neutrino sector. In particular, the ratio $\left(m_{\tilde{l}_{L}}^{2}\right)_{12} /\left(m_{\tilde{l}_{L}}^{2}\right)_{23}$ is highly model dependent while $\left(m_{\tilde{l}_{L}}^{2}\right)_{12} \simeq\left(m_{\tilde{l}_{L}}^{2}\right)_{13}$ to a good approximation [37. The situation is completely different in a type-II see-saw scenario where the mixing angles of $\left(m_{\tilde{l}_{L}}^{2}\right)_{i \neq j}$ are entirely governed by the PMNS matrix and low energy LFV processes relative to different family transitions are strikingly correlated [38].

Furthermore, we remind that a successful Yukawa coupling unification requires either the introduction of some GUT breaking effects or some new non-renormalizable contributions. In both cases, we are forced to introduce a relative rotation matrix $V^{(q l)}$ between the quark and leptonic fields (defined in the super-CKM basis) such 
that the quark-lepton correlations might be modified [39]. However, since the matrix $V^{(q l)}$ is unknown, in the following, we assume the case where the naive quark-lepton correlations are still valid.

\section{HADRONIC SECTOR}

In what follows, we discuss the relevant observables for our study, including $\Delta F=2,1,0$ transitions.

1. The complete set of operators for $\Delta B=2$ transitions is 40 .

$$
\begin{aligned}
Q_{1}^{V L L} & =\left(\bar{b}_{L} \gamma_{\mu} q_{L}\right)\left(\bar{b}_{L} \gamma^{\mu} q_{L}\right), \\
Q_{1}^{S L L} & =\left(\bar{b}_{R} q_{L}\right)\left(\bar{b}_{R} q_{L}\right), \\
Q_{2}^{S L L} & =\left(\bar{b}_{R} \sigma_{\mu \nu} q_{L}\right)\left(\bar{b}_{R} \sigma^{\mu \nu} q_{L}\right), \\
Q_{1}^{L R} & =\left(\bar{b}_{L} \gamma_{\mu} q_{L}\right)\left(\bar{b}_{R} \gamma^{\mu} q_{R}\right), \\
Q_{2}^{L R} & =\left(\bar{b}_{R} q_{L}\right)\left(\bar{b}_{L} q_{R}\right),
\end{aligned}
$$

where $q=s, d, \sigma_{\mu \nu}=\frac{1}{2}\left[\gamma_{\mu}, \gamma_{\nu}\right]$. In Eq. (9) we did not show the operators $Q_{1}^{\mathrm{VRR}}$ and $Q_{1,2}^{S R R}$ that are obtainable from $Q_{1}^{\mathrm{VLL}}$ and $Q_{1,2}^{S L L}$, respectively, with the exchange $q_{L} \rightarrow q_{R}$.

The low energy effective Hamiltonian reads [40]

$$
\mathcal{H}_{\mathrm{eff}}=\sum_{i, a} C_{i}^{a}\left(\mu_{B}, B\right) Q_{i}^{a},
$$

where the summation is performed over contributing operators. The off-diagonal element in the $B_{q}^{0}$-meson mixing is given by

$$
M_{12}^{q}=\frac{1}{3} M_{B_{q}} F_{B_{q}}^{2} \sum_{i, a} C_{i}^{a *}\left(\mu_{H}, B_{q}\right) P_{i}^{a}\left(B_{q}\right),
$$

where $P_{i}^{a}\left(B_{q}^{0}\right)$ collect all RG effects from the high scale, where heavy degrees of freedom are integrated out, down to the $B$ meson scale as well as hadronic matrix elements obtained by lattice QCD techniques. Updating the results of Ref. [40, it turns out that $P_{2}^{L R}\left(B_{q}^{0}\right) \approx 3.4$ and $P_{1}^{S L L}\left(B_{q}^{0}\right) \approx-1.4$ for $\mu_{H}=246 \mathrm{GeV}$.

Introducing the notation

$$
M_{12}^{q}=\left(M_{12}^{q}\right)_{\mathrm{SM}} C_{B_{q}} e^{2 i \varphi_{B_{q}}}, \quad(q=d, s),
$$

the $B_{s, d}^{0}$ mass differences and the CP asymmetries $S_{\psi K_{S}}$ and $S_{\psi \phi}$ are

$$
\begin{aligned}
\Delta M_{q} & =2\left|M_{12}^{q}\right|=\left(\Delta M_{q}\right)_{\mathrm{SM}} C_{B_{q}} \\
S_{\psi K_{S}} & =\sin \left(2 \beta+2 \varphi_{B_{d}}\right) \\
S_{\psi \phi} & =\sin \left(2\left|\beta_{s}\right|-2 \varphi_{B_{s}}\right)
\end{aligned}
$$

where $\sin (2 \beta)_{\text {tree }}=0.734 \pm 0.038$ [32] and $\sin \left(2 \beta_{s}\right)=$ $0.038 \pm 0.003$ [32].

The $\Delta S=2$ operators are obtained from Eq. 9 by means of $b \rightarrow s$ and $q=d$. The off-diagonal element in $K^{0}-\bar{K}^{0}$ mixing $M_{12}^{K}$ is then given by

$$
2 M_{K} M_{12}^{K}=\left\langle\bar{K}^{0}\left|\mathcal{H}_{\mathrm{eff}}\right| K^{0}\right\rangle^{*}
$$

and the observables $\Delta M_{K}$ and $\epsilon_{K}$ can be evaluated through

$$
\begin{aligned}
\Delta M_{K} & =2 \operatorname{Re}\left(M_{12}^{K}\right), \\
\epsilon_{K} & =e^{i \varphi_{\epsilon}} \frac{\kappa_{\epsilon}}{\sqrt{2} \Delta M_{K}} \operatorname{Im}\left(M_{12}^{K}\right),
\end{aligned}
$$

where $\kappa_{\varepsilon}=0.94 \pm 0.02$ [9, 22] accounts for $\varphi_{\varepsilon}=(43.51 \pm$ $0.05)^{\circ} \neq \pi / 4$ and includes long distance contributions.

Let us discuss now the semileptonic asymmetry in $B^{0}$ decays $A_{\mathrm{SL}}^{b}$, described by the quantity [14]

$$
A_{\mathrm{SL}}^{b}=(0.506 \pm 0.043) A_{\mathrm{SL}}^{d}+(0.494 \pm 0.043) A_{\mathrm{SL}}^{s},
$$

where $A_{\mathrm{SL}}^{d}$ and $A_{\mathrm{SL}}^{s}$ are the asymmetries in $B_{d}^{0}$ and $B_{s}^{0}$ decays, respectively. Within the $\mathrm{SM}$, it is predicted that $A_{\mathrm{SL}}^{d}(\mathrm{SM})=\left(-0.48_{-0.12}^{+0.1}\right) \times 10^{-3}[41], A_{\mathrm{SL}}^{s}(\mathrm{SM})=(2.1 \pm$ $0.6) \times 10^{-5}$ [41] and therefore [41]

$$
A_{\mathrm{SL}}^{b}(\mathrm{SM})=\left(-0.23_{-0.06}^{+0.05}\right) \times 10^{-3} .
$$

This has to be compared with the recently measured value by the D0 collaboration [14]

$$
A_{\mathrm{SL}}^{b}(\mathrm{D} 0)=(-9.57 \pm 2.51 \pm 1.46) \times 10^{-3},
$$

that differs by 3.2 standard deviations from the SM prediction, providing the first evidence for anomalous CPviolation in the mixing of neutral $B^{0}$ mesons.

In the presence of NP, the asymmetries $A_{\mathrm{SL}}^{d}$ and $A_{\mathrm{SL}}^{s}$ can be evaluated by means of the following expression [4]

$$
A_{\mathrm{SL}}^{q}=\operatorname{Im}\left(\frac{\Gamma_{12}^{q}}{M_{12}^{q}}\right)^{\mathrm{SM}} \frac{\cos 2 \varphi_{B_{q}}}{C_{B_{q}}}-\operatorname{Re}\left(\frac{\Gamma_{12}^{q}}{M_{12}^{q}}\right)^{\mathrm{SM}} \frac{\sin 2 \varphi_{B_{q}}}{C_{B_{q}}}
$$

where the updated values for $\operatorname{Re}\left(\Gamma_{12}^{q} / M_{12}^{q}\right)^{\mathrm{SM}}$ and $\operatorname{Im}\left(\Gamma_{12}^{q} / M_{12}^{q}\right)^{\mathrm{SM}}$ can be found in [41. Moreover, we recall that $A_{\mathrm{SL}}^{s}$, in the presence of $\mathrm{NP}$ and neglecting $\beta_{s}$, is correlated model-independently with $S_{\psi \phi}$ as $A_{\mathrm{SL}}^{s} \simeq S_{\psi \phi} \times\left(\mathrm{R}_{12}^{s} / C_{B_{s}}\right)$ 42] (for an alternative modelindependent formula, see [43]).

On general grounds, we observe that the $A_{\mathrm{SL}}^{d}$ contribution to $A_{\mathrm{SL}}^{b}$ is constrained model-independently by the limited NP room left to $S_{\psi K_{S}}$. Furthermore, since in the $S S U(5)_{R N}$ model $\left(m_{\tilde{l}_{L}}^{2}\right)_{12} \simeq\left(m_{\tilde{l}_{L}}^{2}\right)_{13}$, implying that $\left(m_{\tilde{d}_{R}}^{2}\right)_{12} \simeq\left(m_{\tilde{d}_{R}}^{2}\right)_{13}$, it turns out that $S_{\psi K_{S}}$ is SM-like to a very good extent after imposing the experimental bound on $\epsilon_{K}$. As a result we conclude that, within the $S S U(5)_{R N}$ model, the NP contributions to $A_{\mathrm{SL}}^{d}$ are completely negligible. By contrast, the NP contributions to $A_{\mathrm{SL}}^{s}$ might be large since in this case the limits from $S_{\psi K_{S}}$ and $\epsilon_{K}$ do not generally apply.

Therefore, within the $S S U(5)_{R N}$ model, it turns out that

$$
A_{\mathrm{SL}}^{b} \approx 0.5 A_{\mathrm{SL}}^{s} \approx-10^{-3} \frac{S_{\psi \phi}}{C_{B_{s}}}
$$

where $S_{\psi \phi} \lesssim 1$ and therefore $A_{\mathrm{SL}}^{b} \gtrsim-10^{-3}$. 
We briefly recall now the leading SUSY contributions to the $K^{0}, B_{d}^{0}$ and $B_{s}^{0}$ mixing amplitudes coming from gluino/squark boxes and, in the large $\tan \beta$ regime, from double Higgs penguin contributions (we refer to ref. [19] and therein references for the full expressions). The dominant gluino/squark boxes read

$$
\left(C_{2}^{L R}\right)_{\tilde{g}} \simeq-\frac{\alpha_{s}^{2}}{m_{\tilde{q}}^{2}}\left[\left(\delta_{d}^{L L}\right)_{i j}\left(\delta_{d}^{R R}\right)_{i j}\right] g_{4}^{(1)}\left(x_{g}\right)
$$

where $\left(\delta_{d}^{L L}\right)_{i j},\left(\delta_{d}^{R R}\right)_{i j}$ are the mass insertion (MI) parameters (as defined in ref. [19]), $x_{g}=M_{\tilde{g}}^{2} / m_{\tilde{q}}^{2}$, the loop function is such that $g_{4}^{(1)}(1)=23 / 180$ and $i j=23,13,12$ for the $B_{s}^{0}, B_{d}^{0}$ and $K^{0}$ systems, respectively.

The dominant Higgs mediated contributions for the $B_{s}^{0}$ system read

$$
\begin{aligned}
\left(C_{2}^{L R}\right)_{H} & \simeq \frac{\alpha_{2}^{2} \alpha_{s}}{4 \pi} \frac{m_{b}^{2} m_{t}^{2}}{2 M_{W}^{4}} \frac{t_{\beta}^{4}}{\left(1+\epsilon t_{\beta}\right)^{4}} \frac{|\mu|^{2}}{M_{A}^{2} m_{\tilde{q}}^{2}} \\
& \times\left[\frac{A_{t}^{*} M_{\tilde{g}}}{m_{\tilde{q}}^{2}} h_{1}\left(x_{g}\right) h_{3}\left(x_{\mu}\right)\right]\left(\delta_{d}^{R R}\right)_{23} V_{t s}
\end{aligned}
$$

where $t_{\beta}=\tan \beta, x_{\mu}=|\mu|^{2} / m_{\tilde{q}}^{2}, h_{1}(1)=4 / 9, h_{3}(1)=$ $-1 / 4,|\epsilon| \approx 10^{-2}$ is the well known resummation factor stemming from ( $t_{\beta}$ enhanced) non-holomorphic threshold corrections and $m_{b}$ has to be evaluated at the scale $M_{A}$. Notice that, the presence of the RR MIs is crucial to avoid the typical $m_{s} / m_{b}$ suppression of $\left(C_{2}^{L R}\right)_{H}$ arising within a MFV scenario 44. The Wilson coefficient for $B_{d}^{0}$ mixing can be obtained by replacing 23 with 13 and $V_{t s}$ by $V_{t d}$.

In the case of $K^{0}$ mixing, the most relevant effect from the neutral Higgses arises only at the fourth order in the MI expansion. Since in the $S S U(5)_{R N}$ model the MI are radiatively induced, this effect can be safely neglected.

Moreover, we remind that in contrast to the case with gluino box contributions, there are no analogous Higgs mediated contributions for $D^{0}-\bar{D}^{0}$ mixing as there are no $\tan \beta$ enhanced non-holomorphic threshold corrections in the up-quark sector.

2. The CP asymmetries $S_{f}$ in the decays of neutral $B_{d}^{0}$ mesons into final CP eigenstates $f$ can be affected by $\mathrm{NP}$ both in the $B_{d}^{0}$ mixing amplitude and the decay amplitudes $\bar{b} \rightarrow \bar{q} q \bar{s}(q=s, d, u)$. In the $S S U(5)_{R N}$ model, only the latter effect can be sizable. The asymmetries are defined as

$$
S_{f}=\frac{2 \operatorname{Im}\left(\lambda_{f}\right)}{1+\left|\lambda_{f}\right|^{2}} .
$$

where $\lambda_{f}=e^{-2 i\left(\beta+\varphi_{B_{d}}\right)}\left(\bar{A}_{f} / A_{f}\right)$ with $\varphi_{B_{d}}$ being the NP phase of the $B_{d}^{0}$ mixing amplitude, $M_{12}^{d}$, and $A_{f}\left(\bar{A}_{f}\right)$ is the decay amplitude for $B_{d}^{0}\left(\overline{B_{d}^{0}}\right) \rightarrow f$. Within the SM it turns out that $A_{f}^{\mathrm{SM}}=A_{f}^{c}\left(1+a_{f}^{u} e^{i \gamma}\right)$ where the $a_{f}^{u}$ parameters have been evaluated in the QCD factorization approach at the leading order and to zeroth order in
$\Lambda / m_{b}$ in 45]. In the presence of NP one can generally define the amplitude $A_{f}$ as

$$
A_{f}=A_{f}^{\mathrm{SM}}+A_{f}^{c} \sum_{i}\left(b_{f i}^{c}+b_{f i}^{u} e^{i \gamma}\right)\left(C_{i}^{*}+\zeta \tilde{C}_{i}^{*}\right),
$$

where $C_{i}$ and $\tilde{C}_{i}$ are the NP contributions to the Wilson coefficients evaluated at the scale $M_{W}$ (see Ref. [19] for the notation), the parameters $b_{f i}^{u}$ and $b_{f i}^{c}$ calculated in [45] and $\zeta= \pm 1$ depending on the parity of the final state; for instance $\zeta=1$ for $\phi K_{S}$ and $\zeta=-1$ for $\eta^{\prime} K_{S}$. Within the $S S U(5)_{R N}$ model the by far dominant contribution to $A_{f}$ is provided by $\tilde{C}_{8}$ which reads [19]

$$
\frac{4 G_{F}}{\sqrt{2}} \tilde{C}_{8}^{\tilde{g}} \simeq \frac{g_{s}^{2}}{m_{\tilde{q}}^{2}} \frac{M_{\tilde{g}} \mu^{*}}{m_{\tilde{q}}^{2}} \frac{t_{\beta}}{\left(1+\epsilon t_{\beta}\right)} \frac{\left(\delta_{d}^{R R}\right)_{32}}{V_{t s}^{*}} g_{8}\left(x_{g}\right) .
$$

where the loop function is such that $g_{8}(1)=-7 / 120$.

The branching ratio for $B_{q} \rightarrow \mu^{+} \mu^{-}$(with $q=s, d$ ) in the presence of NP scalar currents can be expressed as 19

$$
\frac{\mathrm{BR}\left(B_{q} \rightarrow \mu^{+} \mu^{-}\right)}{\operatorname{BR}\left(B_{q} \rightarrow \mu^{+} \mu^{-}\right)_{\mathrm{SM}}} \simeq\left|1-C_{S}^{q}-\tilde{C}_{S}^{q}\right|^{2}+\left|C_{S}^{q}-\tilde{C}_{S}^{q}\right|^{2},
$$

where the relevant contributions to the Wilson coefficients $C_{S}^{q}$ and $\tilde{C}_{S}^{q}$ arising in the $S S U(5)_{R N}$ model read

$$
\begin{aligned}
\tilde{C}_{S}^{q} & \simeq \frac{\alpha_{2} \alpha_{s}}{8 M_{A}^{2}} \frac{m_{B_{q}}^{2}}{M_{W}^{2} C_{10}^{\mathrm{SM}}} \frac{t_{\beta}^{3}}{\left(1+\epsilon t_{\beta}\right)^{2}} \frac{M_{\tilde{g}} \mu^{*}}{m_{\tilde{q}}^{2}}\left(\delta_{d}^{R R}\right)_{3 q} h_{1}\left(x_{g}\right), \\
C_{S}^{q} & \simeq-\frac{\alpha_{2}^{2}}{8 M_{A}^{2}} \frac{m_{B_{q}}^{2}}{M_{W}^{4} C_{10}^{\mathrm{SM}}} \frac{m_{t}^{2} t_{\beta}^{3}}{\left(1+\epsilon t_{\beta}\right)^{2}} \frac{A_{t} \mu}{m_{\tilde{q}}^{2}} V_{t q}^{*} h_{3}\left(x_{\mu}\right),
\end{aligned}
$$

with $C_{10}^{\mathrm{SM}}$ given for instance in [19.

3. Also the hadronic and leptonic EDMs might be generated by flavor dependent phases (flavored EDMs) [46]. In particular, the neutron EDM $d_{n}$ can be estimated from the naive quark model as $d_{n} \approx \frac{4}{3} d_{d}-\frac{1}{3} d_{u}$ (with $d_{f}^{(c)}$ evaluated at $1 \mathrm{GeV}$ ) or, alternatively, by means of QCD sum rule techniques [47 50]. In the latter case, it turns out that

$$
d_{n}=(1 \pm 0.5)\left[1.4\left(d_{d}-0.25 d_{u}\right)+1.1 e\left(d_{d}^{c}+0.5 d_{u}^{c}\right)\right] .
$$

Similarly, the prediction for the Mercury EDM in the QCD sum rule approach reads [49, 50]

$$
d_{\mathrm{Hg}} \simeq 7 \times 10^{-3} e\left(d_{u}^{c}-d_{d}^{c}\right),
$$

where, in eq. (32), we have retained only the contributions relevant to our analysis. Notice that, the values of $d_{f}^{(c)}$ entering the EDM's predictions of eqs. 31 32 32 are assumed to be evaluated at $1 \mathrm{GeV}$ by means of QCD renormalization group evolution [51] from the corresponding values at the electroweak scale. 
The dominant gluino/squark contribution to the downquark (C)EDMs at the SUSY scale reads

$$
\left\{\frac{d_{d_{i}}}{e}, d_{d_{i}}^{c}\right\} \approx \frac{\alpha_{s}}{4 \pi} \frac{m_{b}}{m_{\tilde{q}}^{2}} \frac{M_{\tilde{g}} \mu}{m_{\tilde{q}}^{2}} t_{\beta} \operatorname{Im}\left[\left(\delta_{d}^{L L}\right)_{i 3}\left(\delta_{d}^{R R}\right)_{3 i}\right] f\left(x_{g}\right),
$$

where the loop functions satisfy $f(1)=\{4 / 135,11 / 180\}$.

\section{LEPTONIC SECTOR}

The branching ratio for $\ell_{i} \rightarrow \ell_{j} \gamma$ can be written as

$$
\frac{\operatorname{BR}\left(\ell_{i} \rightarrow \ell_{j} \gamma\right)}{\operatorname{BR}\left(\ell_{i} \rightarrow \ell_{j} \nu_{i} \overline{\nu_{j}}\right)}=\frac{48 \pi^{3} \alpha}{G_{F}^{2}}\left(\left|A_{L}^{i j}\right|^{2}+\left|A_{R}^{i j}\right|^{2}\right) .
$$

Starting from the full expressions of ref. [52] (which we use in our numerical analysis), and specializing to the illustrative case of a degenerate SUSY spectrum with a common mass $m_{\tilde{\ell}}$, one can find

$$
A_{L}^{i j} \simeq \frac{\alpha_{2}}{60 \pi} \frac{t_{\beta}}{m_{\tilde{\ell}}^{2}}\left(\delta_{\ell}^{L L}\right)_{j i} .
$$

The main SUSY contribution to $a_{\mu}^{\mathrm{MSSM}}$ is usually provided by the loop exchange of charginos and sneutrinos. In the limit of degenerate SUSY masses one finds

$$
\frac{a_{\mu}^{\mathrm{MSSM}}}{1 \times 10^{-9}} \approx 1.5\left(\frac{t_{\beta}}{10}\right)\left(\frac{300 \mathrm{GeV}}{m_{\tilde{\ell}}}\right)^{2} \operatorname{sgn} \mu .
$$

Assuming a degenerate SUSY spectrum, it is straightforward to find the correlation between $\Delta a_{\mu}^{\mathrm{SUSY}}$ and the branching ratios for $\ell_{i} \rightarrow \ell_{j} \gamma[37,53]$

$$
\begin{aligned}
& \operatorname{BR}(\mu \rightarrow e \gamma) \approx 2 \times 10^{-12}\left[\frac{\Delta a_{\mu}^{\mathrm{SUSY}}}{3 \times 10^{-9}}\right]^{2}\left|\frac{\left(\delta_{\ell}^{L L}\right)_{21}}{10^{-4}}\right|^{2}, \\
& \operatorname{BR}(\tau \rightarrow \mu \gamma) \approx 8 \times 10^{-8}\left[\frac{\Delta a_{\mu}^{\mathrm{SUSY}}}{3 \times 10^{-9}}\right]^{2}\left|\frac{\left(\delta_{\ell}^{L L}\right)_{32}}{10^{-2}}\right|^{2}
\end{aligned}
$$

where we have assumed that the MIs $\left(\delta_{\ell}^{L L}\right)_{i j}$ provide the dominant contributions to $\operatorname{BR}\left(\ell_{i} \rightarrow \ell_{j} \gamma\right)$, as it happens in the GUT framework analyzed here.

Within the $S S U(5)_{R N}$ model, leptonic EDMs are generated via flavour dependent phases (flavoured EDMs). It turns out that

$$
\frac{d_{\ell_{i}}}{e} \simeq-\frac{\alpha_{Y}}{4 \pi}\left(\frac{m_{\tau}}{m_{\tilde{\ell}}^{2}}\right) t_{\beta} \frac{\operatorname{Im}\left[\left(\delta_{\ell}^{R R}\right)_{i 3}\left(\delta_{\ell}^{L L}\right)_{3 i}\right]}{30}
$$

where a common SUSY mass $m_{\tilde{\ell}}$ has been assumed. If $t_{\beta}=10$ and $m_{\tilde{\ell}}=300 \mathrm{GeV}$, it turns out that $d_{\ell_{i}} \sim$ $10^{-22} \times \operatorname{Im}\left[\left(\delta_{\ell}^{R R}\right)_{i 3}\left(\delta_{\ell}^{L L}\right)_{3 i}\right] e \mathrm{~cm}$.

\section{HADRON-LEPTON CORRELATIONS}

As already anticipated in the Introduction, SUSY GUT models link flavor-violating observables of the leptonic and hadronic sectors. In the following, we provide approximate analytical expressions for these hadronlepton correlations in order to get an idea of where we stand. In particular, the GUT relation $\left(\delta_{e}^{L L}\right)_{i j}=$ $\left(\delta_{d}^{R R}\right)_{j i}$, which is modified at the electroweak scale as $\left(\delta_{e}^{L L}\right)_{i j}=\left(\delta_{d}^{R R}\right)_{j i} \times\left(m_{\tilde{q}} / m_{\tilde{\ell}}\right)^{2}$, implies

$$
\begin{aligned}
\operatorname{BR}(\mu \rightarrow e \gamma) & \approx 2 \times 10^{-12}\left(\frac{\epsilon_{K}^{S U S Y}}{10^{-4}}\right)^{2}\left(\frac{t_{\beta}}{10}\right)^{2} \\
& \times\left|\frac{\left(\delta_{d}^{L L}\right)_{12}}{10^{-4}}\right|^{-2}\left(\frac{m_{\tilde{q}}}{m_{\tilde{\ell}}}\right)^{8},
\end{aligned}
$$

and similarly for the 23 sector

$$
\begin{aligned}
\operatorname{BR}(\tau \rightarrow \mu \gamma) & \approx 7 \times 10^{-8}\left[\left(C_{B_{s}}-1\right)^{2}+C_{B_{s}} S_{\psi \phi}^{2}\right] \\
& \times\left(\frac{t_{\beta}}{10}\right)^{2}\left|\frac{\left(\delta_{d}^{L L}\right)_{23}}{10^{-2}}\right|^{-2}\left(\frac{m_{\tilde{q}}}{m_{\tilde{\ell}}}\right)^{8},
\end{aligned}
$$

where we have assumed a common SUSY mass $m_{\tilde{q}}\left(m_{\tilde{\ell}}\right)$ for the hadron (lepton) sector and $\left(\delta_{d}^{L L}\right)_{12,23}$ have been normalized to the typical values they attain when they are radiatively generated by the large top Yukawa coupling and the CKM matrix (see Eq. (8)).

Finally, there is an even more direct correlation between $\operatorname{BR}(\tau \rightarrow \mu \gamma)$ and the NP effects entering $S_{\phi K_{S}}$. Defining $S_{\phi K_{S}}=S_{\psi K_{S}}+\Delta S_{\phi K_{S}}$, we find

$$
\operatorname{BR}(\tau \rightarrow \mu \gamma) \approx 3 \times 10^{-8}\left(\frac{m_{\tilde{q}}}{m_{\tilde{\ell}}}\right)^{8}\left|\Delta S_{\phi K_{S}}\right|^{2},
$$

where, starting from eqs. (26), (27), (28) and keeping only linear terms in the NP contributions, it turns out that $\Delta S_{\phi K_{S}} \approx-2 b_{\phi K_{S}}^{c} \cos 2 \beta \operatorname{Im} \tilde{C}_{8}$ with $b_{\phi K_{S}}^{c} \approx 1.4$ [45].

\section{NUMERICAL ANALYSIS}

In this section, we present the numerical results for the observables discussed in the previous sections in the context of the $S S U(5)_{R N}$ model, assuming a gravity mediated mechanism for the SUSY breaking terms with $M_{P}=2.4 \times 10^{18} \mathrm{GeV}$.

In the upper (left) plot of Fig. 2, we show the predictions for $\operatorname{BR}(\mu \rightarrow e \gamma)$ vs. $\epsilon_{K}^{S U S Y} /\left(\epsilon_{K}^{S M}\right)$ varying the SUSY parameters in the ranges $\left(m_{0}, M_{1 / 2}\right)<1 \mathrm{TeV}$, $\left|A_{0}\right|<3 m_{0}, \tan \beta=10$ and $\mu>0$. Concerning the neutrino sector, hereafter, we assume a hierarchical spectrum for both light and heavy neutrinos such that $m_{\nu_{3}}=0.05 \mathrm{eV}$ and we vary the neutrino parameters in the ranges $10^{11} \leq M_{\nu_{3}}(\mathrm{GeV}) \leq 10^{15}, 10^{-5} \leq U_{e 3} \leq 0.1$.

The blue (red) points can explain the $(g-2)_{\mu}$ anomaly at the level of $\Delta a_{\mu}^{\text {SUSY }} \gtrsim 1(2) \times 10^{-9}$ while satisfying the 

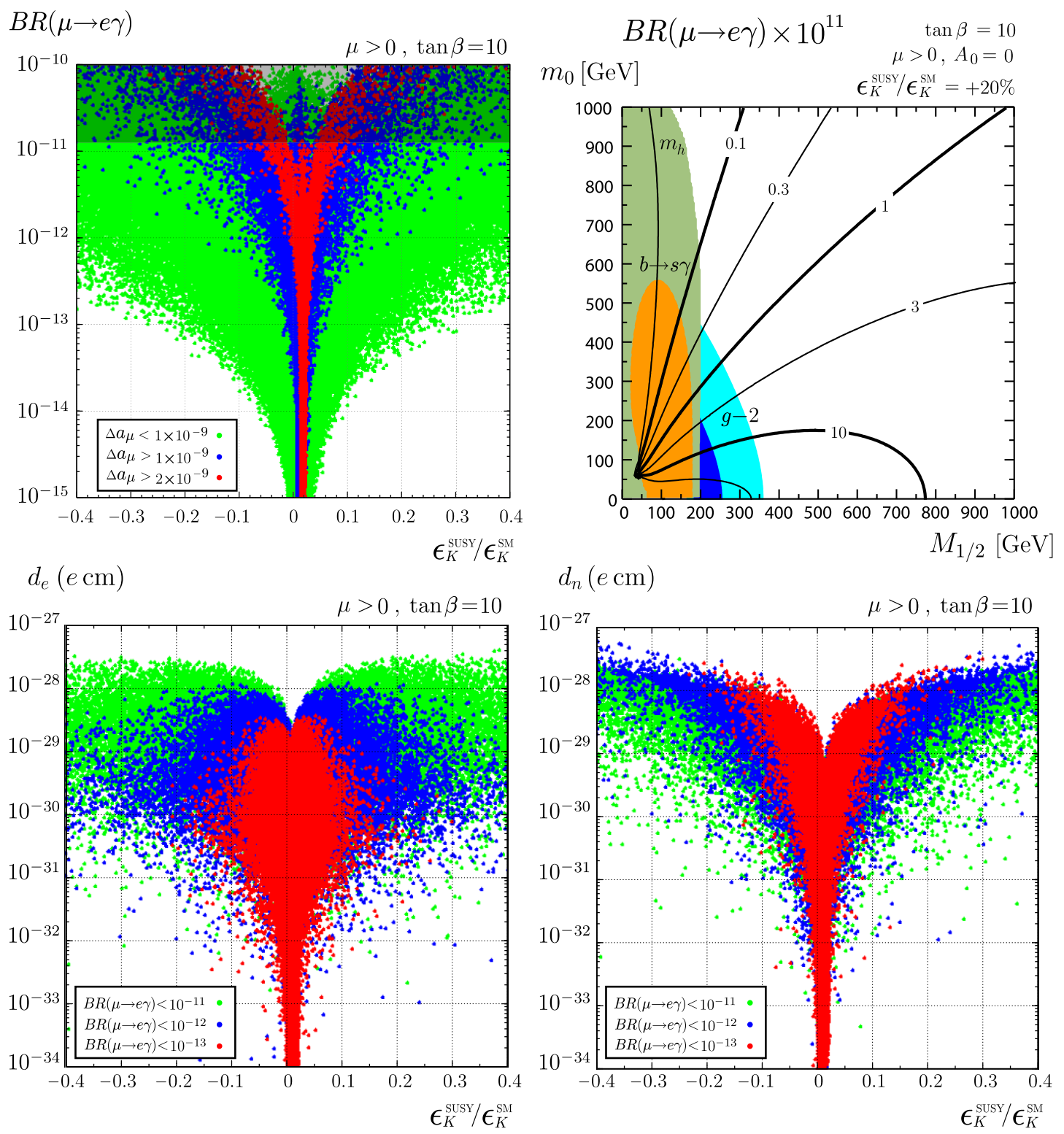

FIG. 2: Upper left: $\operatorname{BR}(\mu \rightarrow e \gamma)$ vs. $\epsilon_{K}^{S U S Y} /\left(\epsilon_{K}^{S M}\right)$. The blue (red) points can explain the $(g-2)_{\mu}$ anomaly at the level of $\Delta a_{\mu}^{\text {SUSY }} \gtrsim 1(2) \times 10^{-9}$. Upper right: $\mathrm{BR}(\mu \rightarrow e \gamma)$ in the $\left(m_{0}, M_{1 / 2}\right)$ plane imposing a $+20 \%$ NP effect in $\epsilon_{K}^{S U S Y}$. Lower left (right): electron (neutron) EDM vs. $\epsilon_{K}^{S U S Y} /\left(\epsilon_{K}^{S M}\right)$ for different values of $\operatorname{BR}(\mu \rightarrow e \gamma)$. In all the plots we vary the SUSY parameters in the ranges $m_{0}<1 \mathrm{TeV}, M_{1 / 2}<1 \mathrm{TeV},\left|A_{0}\right|<3 m_{0}$ (in the only upper right plot we set $\left|A_{0}\right|=0$ ), tan $\beta=10$ and $\mu>0$. We assume a hierarchical spectrum for both light and heavy neutrinos setting $m_{\nu_{3}}=0.05 \mathrm{eV}$ and varying the neutrino parameters in the ranges $10^{11} \leq M_{\nu_{3}}(\mathrm{GeV}) \leq 10^{15}, 10^{-5} \leq U_{e 3} \leq 0.1$.

constraints from $\mathrm{BR}\left(B \rightarrow X_{s} \gamma\right) 27$ at the $99 \%$ C.L.. As we can see, sizable SUSY effects in $\epsilon_{K}$, that might be desirable to solve the UT anomaly, generally imply a lower bound for $\operatorname{BR}(\mu \rightarrow e \gamma)$ in the reach of the MEG experiment. The above statement is even more strengthened if we further require to explain the $(g-2)_{\mu}$ anomaly.

In the upper (right) plot of Fig. 2, we show the values reached by $\operatorname{BR}(\mu \rightarrow e \gamma)$ in the $\left(m_{0}, M_{1 / 2}\right)$ plane setting $\mu>0, A_{0}=0, \tan \beta=10$ and imposing a NP effect in $\epsilon_{K}^{S U S Y}$ at the level of $+20 \%$ compared to the SM contribution to solve the UT tension. The grey region is excluded by the constraint from the lower bound on the lightest Higgs boson mass $m_{h^{0}}$ (we impose $m_{h^{0}}>111.4 \mathrm{GeV}$ to take into account the theoretical uncertainties in the evaluation of $\left.m_{h^{0}}\right)$, the orange region is excluded by the constraints on $\operatorname{BR}\left(B \rightarrow X_{s} \gamma\right)$ at the $99 \%$ C.L. (we have evaluated $\operatorname{BR}\left(B \rightarrow X_{s} \gamma\right)$ including the SM effects at the NNLO 54 and the NP contributions at the LO), the light blue (blue) region satisfies $\Delta a_{\mu}^{\text {SUSY }} \gtrsim 1(2) \times 10^{-9}$.

In the lower plots of Fig. 2 on the left (right), we show the electron (neutron) EDM vs. $\epsilon_{K}^{S U S Y} /\left(\epsilon_{K}^{S M}\right)$ for different values of $\operatorname{BR}(\mu \rightarrow e \gamma)$. The requirement of sizable 

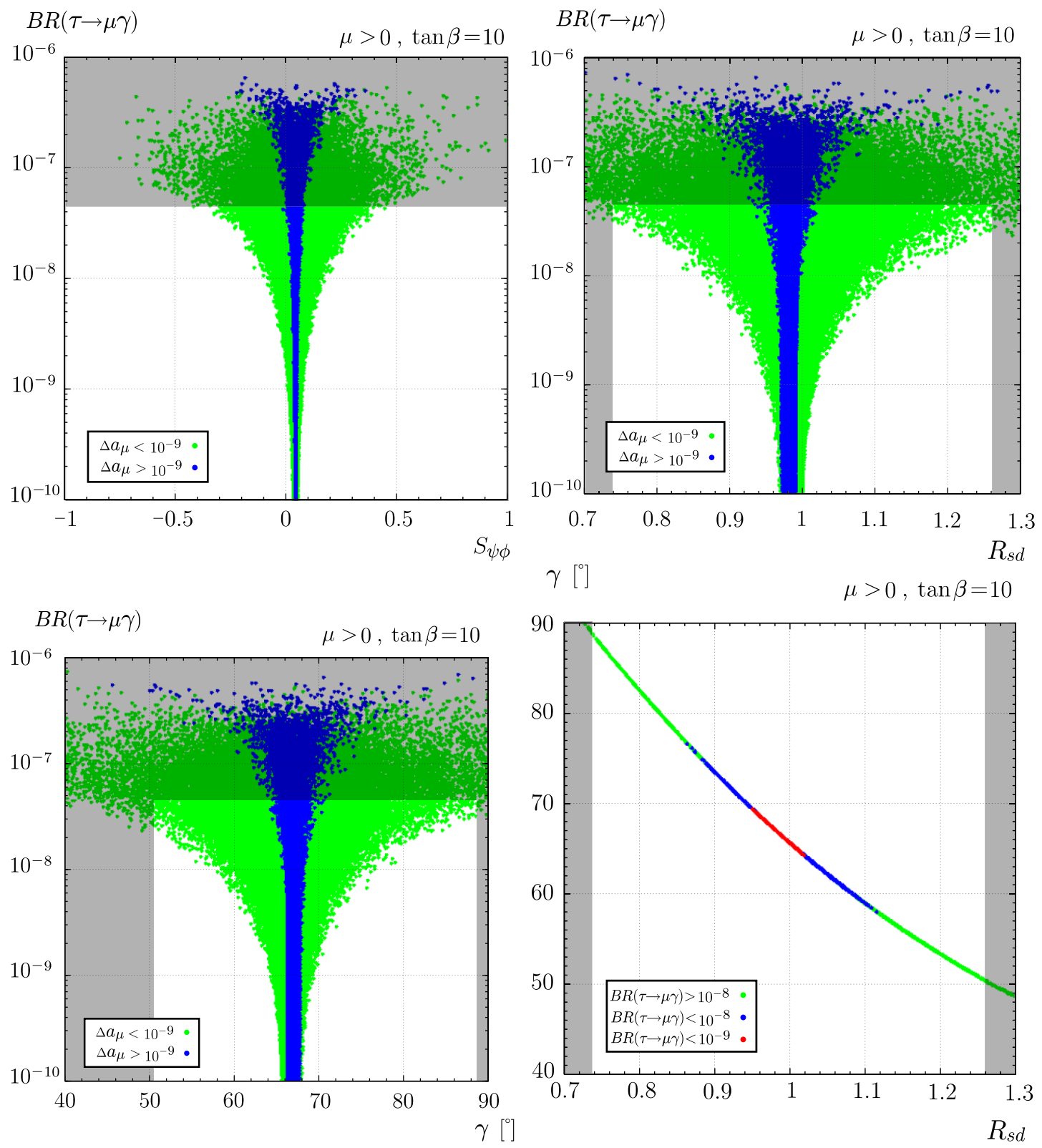

FIG. 3: Upper left: $\operatorname{BR}(\tau \rightarrow \mu \gamma)$ vs. $S_{\psi \phi}$. Upper right: $\operatorname{BR}(\tau \rightarrow \mu \gamma)$ vs. $R_{s d}$. Lower left: $\operatorname{BR}(\tau \rightarrow \mu \gamma)$ vs. $\gamma$. Lower right: $\gamma$ vs. $R_{s d}$. Blue points satisfy the condition $\Delta a_{\mu}^{\text {SUSY }} \gtrsim 1 \times 10^{-9}$ while the grey bands indicate the experimentally excluded regions. In all the plots we vary the SUSY parameters in the ranges $m_{0}<1 \mathrm{TeV}, M_{1 / 2}<1 \mathrm{TeV},\left|A_{0}\right|<3 m_{0}$, tan $\beta=10$ and $\mu>0$. We assume a hierarchical spectrum for both light and heavy neutrinos setting $m_{\nu_{3}}=0.05 \mathrm{eV}, U_{e 3}=0$ and varying the heaviest heavy neutrino mass in the ranges $10^{13} \leq M_{\nu_{3}}(\mathrm{GeV}) \leq 10^{15}$.

non-standard effects in $\epsilon_{K}^{S U S Y}$ always implies large values for $d_{e, n}$, in the reach of the planned experimental resolutions, as well as values for $\operatorname{BR}(\mu \rightarrow e \gamma)$ that are most likely within the MEG reach. The correlations between leptonic and hadronic observables in the plots on the left in Fig. 2 demonstrate very clearly that we deal here with a GUT scenario.

In figs. 3, 4, we present the predictions for B-physics observables. As discussed in the previous sections, within a $S S U(5)_{R N}$ model, $b \rightarrow s$ and $\tau \rightarrow \mu$ transitions are linked, therefore, processes like $B_{s}^{0}$ mixing and $\tau \rightarrow \mu \gamma$ turn out to be related.

In the plots of fig. 3 we vary the SUSY parameters in the ranges $\left(m_{0}, M_{1 / 2}\right)<1 \mathrm{TeV},\left|A_{0}\right|<3 m_{0}, \tan \beta=10$ and $\mu>0$. We assume a hierarchical spectrum for both light and heavy neutrinos setting $m_{\nu_{3}}=0.05 \mathrm{eV}, U_{e 3}=$ 0 and varying the heaviest heavy neutrino mass in the range $10^{13} \leq M_{\nu_{3}}(\mathrm{GeV}) \leq 10^{15}$.

In the upper plot of fig. 3 on the left, we show the correlation between $\operatorname{BR}(\tau \rightarrow \mu \gamma)$ and $S_{\psi \phi}$. We see that, non-standard values for $S_{\psi \phi}$ imply a lower bound for $\mathrm{BR}(\tau \rightarrow \mu \gamma)$ within the SuperB reach. However, it seems 
$B R\left(B_{s} \rightarrow \mu^{+} \mu^{-}\right)$

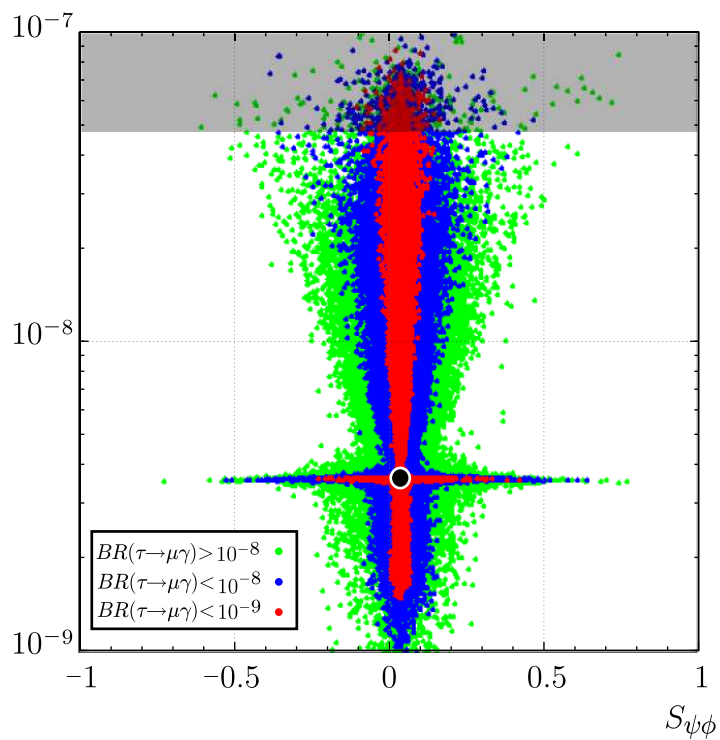

$B R\left(B_{d} \rightarrow \mu^{+} \mu^{-}\right)$
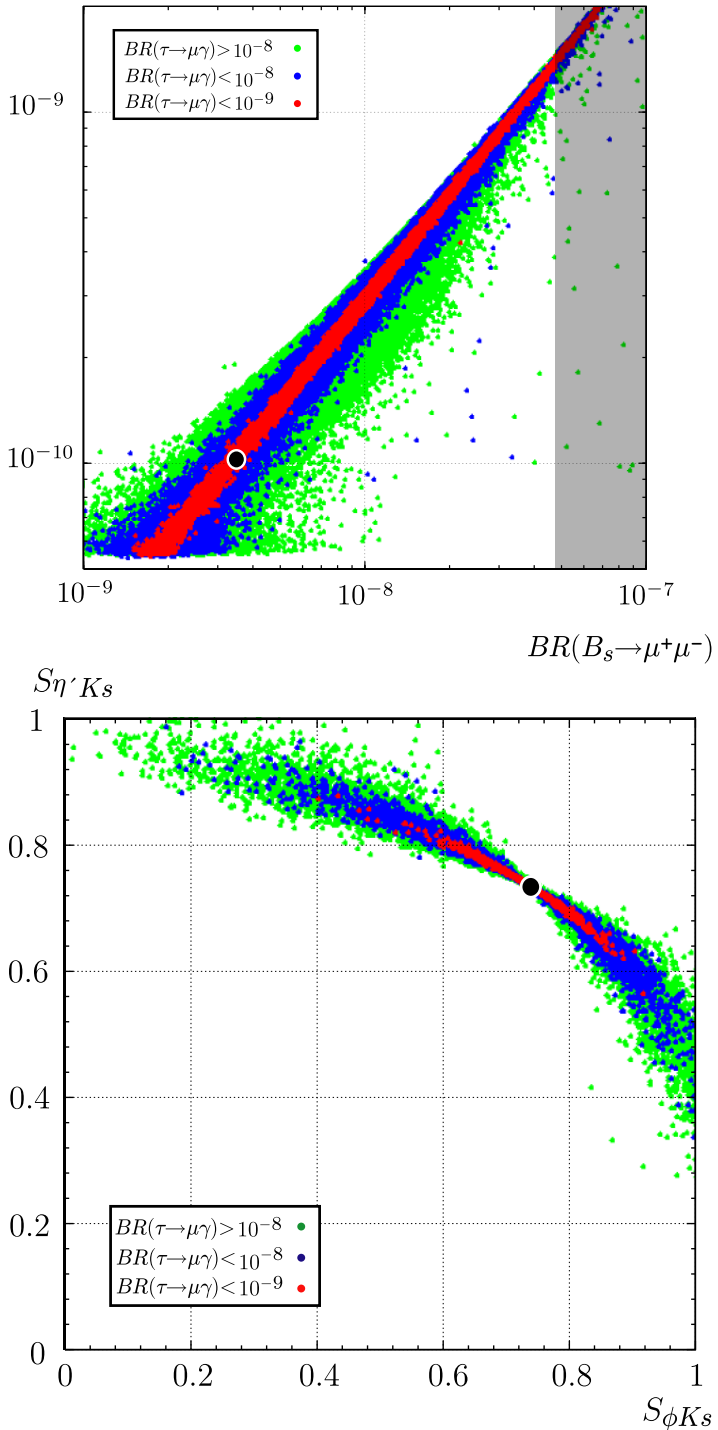

$A_{S L}^{S}$

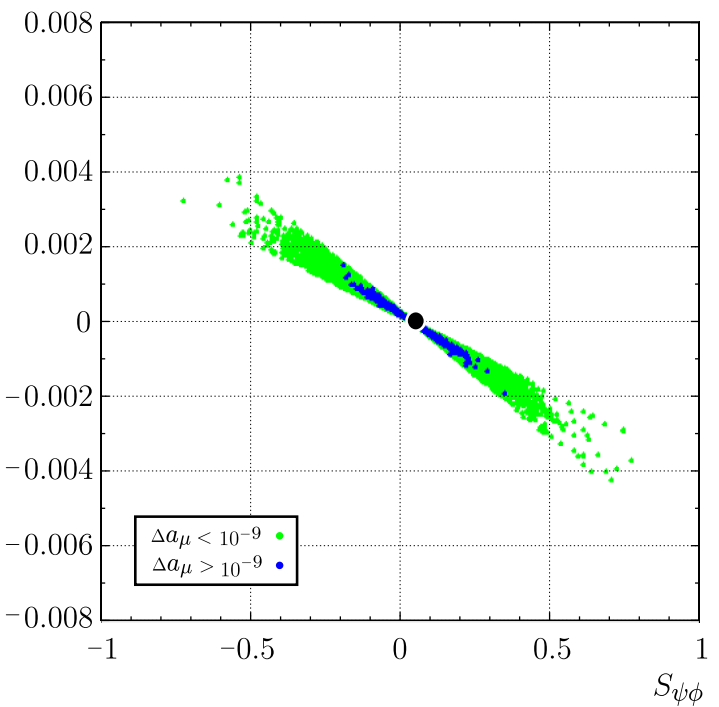

$S_{\phi K s}$

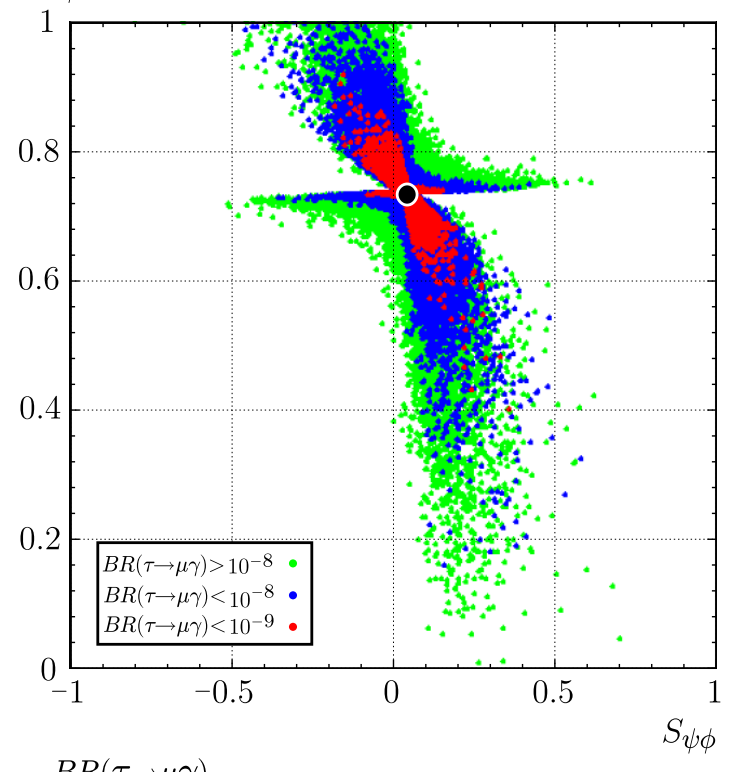

$B R(\tau \rightarrow \mu \gamma)$

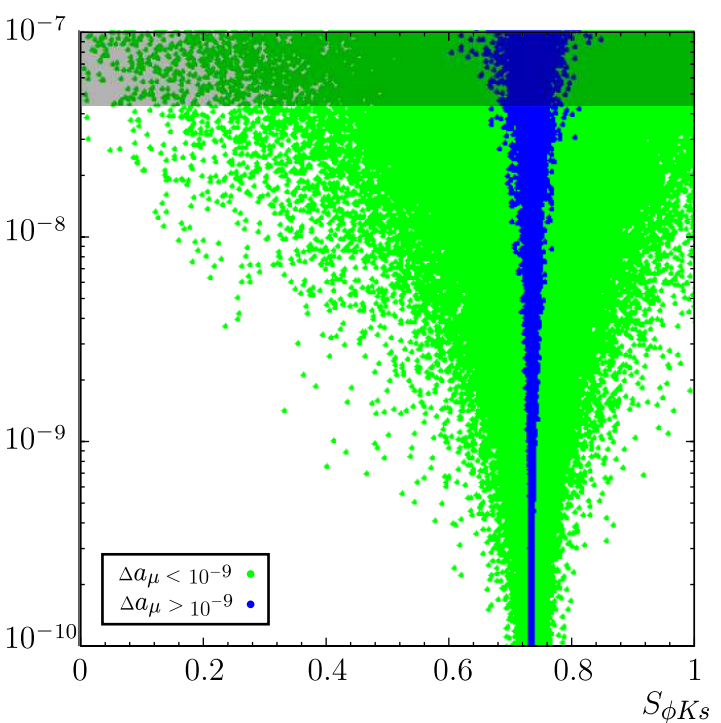

FIG. 4: Upper left: $\operatorname{BR}\left(B_{s} \rightarrow \mu^{+} \mu^{-}\right)$vs. $S_{\psi \phi}$. Upper right: $S_{\psi_{\phi}}$ vs. $A_{S L}^{s}$. Central left: $\operatorname{BR}\left(B_{d} \rightarrow \mu^{+} \mu^{-}\right)$vs. $\operatorname{BR}\left(B_{s} \rightarrow \mu^{+} \mu^{-}\right)$. Central right: $S_{\psi \phi}$ vs. $S_{\phi K_{S}}$. Lower left: $S_{\eta^{\prime} K_{S}}$ vs. $S_{\phi K_{S}}$. Lower right: $\operatorname{BR}(\tau \rightarrow \mu \gamma)$ vs. $S_{\phi K_{S}}$. The grey bands indicate the experimentally excluded regions. In all the plots we vary the SUSY parameters in the ranges $m_{0}<2 \mathrm{TeV}, M_{1 / 2}<1 \mathrm{TeV}$, $\left|A_{0}\right|<3 m_{0}, \tan \beta \leq 60$ and $\mu>0$. We assume a hierarchical spectrum for both light and heavy neutrinos setting $m_{\nu_{3}}=0.05 \mathrm{eV}$, $U_{e 3}=0$ and varying the heaviest heavy neutrino mass in the ranges $10^{13} \leq M_{\nu_{3}}(\mathrm{GeV}) \leq 10^{15}$. 
unlikely to simultaneously explain the $(g-2)_{\mu}$ anomaly (blue points correspond to $\Delta a_{\mu}^{\text {SUSY }} \gtrsim 1 \times 10^{-9}$ ) while generating a large $S_{\psi \phi}$. The situation can slightly change for large values of $\tan \beta$. In this case, the constraints from $\mathrm{BR}(\tau \rightarrow \mu \gamma)$ might be still compatible with $\left|S_{\psi \phi}\right| \leq 0.2$ and $\Delta a_{\mu}^{\text {SUSY }} \gtrsim 1 \times 10^{-9}$ (see fig. 4).

In the upper plot of fig. 3 on the right, we show the correlation between $\operatorname{BR}(\tau \rightarrow \mu \gamma)$ vs. $R_{s d}$ defined as

$$
R_{s d}=\frac{\Delta M_{d} / \Delta M_{s}}{\left(\Delta M_{d} / \Delta M_{s}\right)_{\mathrm{SM}}} .
$$

We see that non-standard effects in $R_{s d}$ may be easily generated, providing a possible solution to the UT anomaly. This will imply in turn a lower bound for $\mathrm{BR}(\tau \rightarrow \mu \gamma)$ within the expected experimental reach of a SuperB.

In the lower plot of fig. 3 on the left, we show the correlation between $\operatorname{BR}(\tau \rightarrow \mu \gamma)$ and the CKM angle $\gamma$. This correlation can be understood looking at the explicit expressions for $\gamma$ and $R_{t}$ in the presence of NP, see eqs. (34). In particular, NP effects in $R_{s d}$ would affect $R_{t}$ and therefore the determination of $\gamma$. Moreover, since $b \rightarrow s$ and $\tau \rightarrow \mu$ transitions are linked in our framework, it turns out that non-standard values for $\gamma$ imply a lower bound for $\operatorname{BR}(\tau \rightarrow \mu \gamma)$. It will be exciting to monitor such a correlated NP effect at the LHCb.

In the lower plot of fig. 3 on the right, we report the correlation between $\gamma$ vs. $R_{s d}$ clearly showing that negative NP effects in $R_{s d}$, accounting for the UT anomaly, would imply large non-standard values for the angle $\gamma$. Such large non-standard effects would also imply large (visible) values for $\operatorname{BR}(\tau \rightarrow \mu \gamma)$ as seen in the plot on the left.

In fig. 4. we show the predictions for B-physics and lepton observables including the large $\tan \beta$ regime, in order to make Higgs mediated effects for $\Delta F=1,2$ processes like $\operatorname{BR}\left(B_{s, d} \rightarrow \mu^{+} \mu^{-}\right)$and $S_{\psi \phi}$ visible.

In particular, the plots of fig. 4 have been obtained employing the following scan over the SUSY parameters: $m_{0}\left(M_{1 / 2}\right)<2(1) \mathrm{TeV},\left|A_{0}\right|<3 m_{0}, \tan \beta<60$ and $\mu>0$. We assume a hierarchical spectrum for both light and heavy neutrinos setting $m_{\nu_{3}}=0.05 \mathrm{eV}, U_{e 3}=0$ and varying the heaviest heavy neutrino mass in the range $10^{13} \leq M_{\nu_{3}}(\mathrm{GeV}) \leq 10^{15}$.

In the upper plot of fig. 4 on the left, we show $\operatorname{BR}\left(B_{s} \rightarrow \mu^{+} \mu^{-}\right)$vs. $S_{\psi \phi}$ visualizing also the values attained by $\operatorname{BR}(\tau \rightarrow \mu \gamma)$ with different colours. As we can see, $S_{\psi \phi}$ can depart from the SM expectations irrespective of whether $\operatorname{BR}\left(B_{s} \rightarrow \mu^{+} \mu^{-}\right)$is SM-like or not. The reason is that $S_{\psi \phi}$ receives large effects from both gluino/squark box contributions and from $\tan \beta$ enhanced double penguin Higgs contributions. In contrast, in the case of $\operatorname{BR}\left(B_{s} \rightarrow \mu^{+} \mu^{-}\right)$only the latter contribution can be effective.

In the upper plot of fig. 4 on the right, we show the (almost) model-independent correlation between $A_{S L}^{s}$ and $S_{\psi \phi}$ (see eq. (23)). Green points fulfill all the current available constraints, while blue points further explain the $(g-2)_{\mu}$ anomaly at the level of $\Delta a_{\mu}^{\mathrm{SUSY}} \gtrsim 1 \times 10^{-9}$. While large departures from the SM expectations for $A_{S L}^{s}$ are still allowed, the large value reported by the Tevatron [14] (see eq. 21) cannot be accounted for within the $S S U(5)_{R N}$ model.

In the central plot of fig. 4 on the left, we show the correlation between $\operatorname{BR}\left(B_{s} \rightarrow \mu^{+} \mu^{-}\right)$and $\operatorname{BR}\left(B_{d} \rightarrow\right.$ $\left.\mu^{+} \mu^{-}\right)$. Interestingly enough, we notice that sizable departures from the MFV predictions $\left|V_{t s} / V_{t d}\right|^{2}$ imply large values for $\operatorname{BR}(\tau \rightarrow \mu \gamma)$, well within the SuperB reach.

In the central plot of fig. 4 on the right, we show the predictions for $S_{\psi \phi}$ and $S_{\phi K_{S}}$. Noteworthy enough, these observables can sizably depart from the SM expectations in a correlated manner. The pattern of correlation is twofold: for moderate/low $\tan \beta$ values (corresponding to the almost vertical band), $S_{\psi \phi}$ receives the dominant contributions from gluino/squark boxes and the correlation might be in agreement with the current non-standard experimental data while for large $\tan \beta$ values (corresponding to the almost horizontal band), $S_{\psi \phi}$ receives the dominant contributions from double penguin Higgs exchanges and the correlation is opposite. In any case, large non-standard effects for $S_{\psi \phi}$ and/or $S_{\phi K_{S}}$ always imply experimentally visible values for $\operatorname{BR}(\tau \rightarrow \mu \gamma)$.

In the lower plot of fig. 4 on the left, we report the correlation between $S_{\eta^{\prime} K_{S}}$ and $S_{\phi K_{S}}$ clearly showing that these observables exhibit opposite deviations with respect to the SM expectations.

This is understood remembering that the NP amplitudes for these processes can be written as $A_{\mathrm{NP}} \sim$ $C_{i}+\zeta \tilde{C}_{i}$ where $C_{i}$ and $\tilde{C}_{i}$ are the NP Wilson coefficients and $\zeta= \pm 1$ depending on the parity of the final state which is $\zeta=1$ for $\phi K_{S}$ and $\zeta=-1$ for $\eta^{\prime} K_{S}$ (see Section 4). Since in the $S S U(5)_{R N}$ model $\tilde{C}_{i}$ provide the largely dominant effects, $S_{\eta^{\prime} K_{S}}$ and $S_{\phi K_{S}}$ are expected to show opposite departures from the SM predictions as confirmed numerically. This is in contrast to scenarios like the flavour-blind MSSM [55], the MSSM with MFV or models with purely left-handed currents where $C_{i}$ are dominant 19 .

In the lower plot of fig. 4 on the right, we also show the correlation between $\operatorname{BR}(\tau \rightarrow \mu \gamma)$ and $S_{\phi K_{S}}$ confirming that sizable NP effects for $S_{\phi K_{S}}$ imply a lower bound for $\operatorname{BR}(\tau \rightarrow \mu \gamma)$ within the SuperB reach. However, we notice that an explanation of the $(g-2)_{\mu}$ anomaly would prevent large non-standard effects for $S_{\phi K_{S}}$.

\section{DNA-FLAVOUR TEST OF $S S U(5)_{\mathrm{RN}}$}

The pattern of flavour violation predicted by specific NP model represents one of the most powerful tools in the attempt to probe or to falsify the model in question. Motivated by this consideration, in Ref. [19] a "DNAFlavour Test" has been introduced with the aim of summarizing the potential size of deviations from the SM results for the most interesting observables in a selection of SUSY and non-SUSY models. 
In tab. II. we extend such a "DNA-Flavour Test" to the $S S U(5)_{R N}$ model. We remind that we distinguish among large, moderate (but still visible) and vanishingly small effects by three red stars, two blue stars and one black star, respectively.

While we refer to Ref. [19] for a detailed description of the pattern of NP effects in various SUSY models, we want to comment here about one of the most remarkable difference we found between the $S S U(5)_{R N}$ model and the SUSY flavour models discussed in Ref. [19].

In fact, none of the models discussed in Ref. [19] was able to simultaneously account for the current data for $S_{\psi \phi}$ and $S_{\phi K_{S}}$, in contrast to the $S S U(5)_{R N}$ model discussed here.

The reason for this can be traced back recalling that $S_{\phi K_{S}}$ receives the dominant effects from gluino/squark penguins while $S_{\psi \phi}$ either from gluino/squark boxes (at moderate/low $\tan \beta$ values) or from double Higgs penguins (at large $\tan \beta$ ). However, only the moderate/low $\tan \beta$ solution can simultaneously account for an enhancement of $S_{\psi \phi}$ and a suppression of $S_{\phi K_{S}}$ (relative to $S_{\psi K_{S}}$ ) as required by the data. Yet such effects are strongly constrained either by $D^{0}-\bar{D}^{0}$ mixing (in case of Abelian flavour models) or by $K^{0}-\bar{K}^{0}$ mixing (in case of non-Abelian flavour models). Consequently, in this region of parameter space $S_{\psi \phi}$ cannot be large in these models. In fact $S_{\psi \phi}$ receives in these models large values only at large $\tan \beta$ where the sign of the correlation between $S_{\psi \phi}$ and $S_{\phi K_{S}}$ is found to be opposite to data [19], that is $S_{\phi K_{S}}$ is enhanced rather than suppressed when $S_{\psi \phi}$ is enhanced.

In contrast, the $S S U(5)_{R N}$ model predicts unobservable effects for $D^{0}-\bar{D}^{0}$ mixing while the NP effects in $K^{0}-\bar{K}^{0}$ are generally unrelated to those entering $B_{s}^{0}-\bar{B}_{s}^{0}$ mixing and therefore the tight bounds from $\epsilon_{K}$ can be always avoided. Therefore at moderate/low $\tan \beta$ the suppression of $S_{\phi K_{S}}$ and simultaneous sizable enhancement of $S_{\psi \phi}$ can be obtained.

In this context let us recall that within the SM4, the SM with fourth sequential generation, the correlation between $S_{\psi \phi}$ and in $S_{\phi K_{S}}$ is qualitatively similar to the one found in the $S S U(5)_{R N}$ model, that is with increasing $S_{\psi \phi}$ the asymmetry $S_{\phi K_{S}}$ decreases in accordance with the data [56 58. However, in this model the absence of right-handed currents implies, in contrast to $S S U(5)_{R N}$, that also $S_{\eta^{\prime} K_{S}}$ decreases with increasing $S_{\psi \phi}$.

Finally, it has to be stressed that the "DNA-Flavour Test" table doesn't account for possible correlations among observables. Therefore, since simultaneous large effects are not always possible for certain sets of observables, it will be interesting to monitor the changes in this table with improved experimental results.

\section{CONCLUSIONS}

Despite of the remarkable agreement of flavour data with the SM predictions in the $K$ and $B_{d}$ systems, a

\begin{tabular}{|l|c|}
\hline Observable & $S S U(5)_{\mathrm{RN}}$ model \\
\hline \hline$D^{0}-\bar{D}^{0}$ & $\star$ \\
\hline$\epsilon_{K}$ & $\star \star \star$ \\
\hline$S_{\psi K_{S}}$ & $\star \star$ \\
\hline$\gamma$ & $\star \star \star$ \\
\hline$R_{t}$ & $\star \star \star$ \\
\hline$S_{\psi \phi}$ & $\star \star \star$ \\
\hline \hline$S_{\phi K_{S}}$ & $\star \star$ \\
\hline$S_{\eta^{\prime} K_{S}}$ & $\star$ \\
\hline$A_{\mathrm{CP}}\left(B \rightarrow X_{s} \gamma\right)$ & $\star$ \\
\hline$A_{7,8}\left(B \rightarrow K^{*} \mu^{+} \mu^{-}\right)$ & $\star$ \\
\hline$A_{9}\left(B \rightarrow K^{*} \mu^{+} \mu^{-}\right)$ & $\star \star \star$ \\
\hline$B \rightarrow K^{(*)} \nu \bar{\nu}$ & $\star$ \\
\hline$B_{s} \rightarrow \mu^{+} \mu^{-}$ & $\star$ \\
\hline$K^{+} \rightarrow \pi^{+} \nu \bar{\nu}$ & $\star \star \star$ \\
\hline$K_{L} \rightarrow \pi^{0} \nu \bar{\nu}$ & $\star \star \star$ \\
\hline$\mu \rightarrow e \gamma$ & $\star \star \star$ \\
\hline$\tau \rightarrow \mu \gamma$ & $\star \star \star$ \\
\hline$\mu+N \rightarrow e+N$ & $\star \star \star$ \\
\hline \hline$d_{n}$ & $\star \star \star$ \\
\hline$d_{H g}$ & \\
\hline$d_{e}$ & $\star$ \\
\hline$(g-2)_{\mu}$ & $\star$ \\
\hline
\end{tabular}

TABLE II: "DNA" of flavour physics effects for the $S S U(5)_{R N}$ model. $\star \star \star \star$ signals large effects, $\star \star$ visible but small effects and $\star$ implies vanishingly small effects.

closer look at the data might indicate some tensions especially in $\mathrm{CP}$ violating observables. In particular, the most recent UT analyses show some tensions at the level of $(2-3) \sigma[11$ and recent messages from the Tevatron seem to hint the presence of new sources of CPV entering the $B_{s}^{0}$ systems 1214 .

Motivated by the above facts, in the present work, we have analyzed the low energy implications of a supersymmetric $S U(5)$ GUT scenario with right-handed neutrinos 17. $\left(S S U(5)_{R N}\right)$ accounting for the neutrino masses and mixing angles by means of a type-I see-saw mechanism [3].

Since supersymmetric Grand Unified theories generally predict $\mathrm{FCNC}$ and $\mathrm{CP}$ violating processes to occur both in the leptonic and hadronic sectors, we have performed an extensive study of FCNC and CP Violation in both sectors, analyzing possible hadron/lepton correlations among observables. In particular, we have monitored the low energy consequences implied by the solutions to the above tensions. 
However, within the $S S U(5)_{R N}$ model, it is not possible to link model independently different flavour transitions like $s \rightarrow d$ and $b \rightarrow s$. In fact, the neutrino Yukawa couplings, which regulate the size of the flavour violation both in the hadronic and leptonic sectors, are unknown. Therefore, we have analyzed the phenomenology related to $s \rightarrow d$ and $b \rightarrow s$ transitions separately.

The main results of our study of the $s \rightarrow d$ transitions and their correlation with $\mu \rightarrow e$ transitions are

- Sizable SUSY effects in $\epsilon_{K}$, that might be desirable to solve the UT anomaly, generally imply a lower bound for $\operatorname{BR}(\mu \rightarrow e \gamma)$ in the reach of the MEG experiment. Furthermore, the simultaneous requirement of an explanation for both the $(g-2)_{\mu}$ and the UT anomalies would typically im$\operatorname{ply} \operatorname{BR}(\mu \rightarrow e \gamma) \geq 10^{-12}$.

- The requirement of sizable non-standard effects in $\epsilon_{K}^{S U S Y}$ always implies large values for the electron and neutron EDMs, in the reach of the planned experimental resolutions.

The main results of our study of the $b \rightarrow s$ transitions and of their correlations with $\tau \rightarrow \mu$ transitions are

- Non-standard values for $S_{\psi \phi}$ imply a lower bound for $\operatorname{BR}(\tau \rightarrow \mu \gamma)$ within the SuperB reach. However, the $(g-2)_{\mu}$ anomaly can be solved only for large $\tan \beta$ values where we find $\left|S_{\psi \phi}\right| \leq 0.2$ for $\Delta a_{\mu}^{\text {SUSY }} \gtrsim 1 \times 10^{-9}$ while being still compatible with the constraints from $\operatorname{BR}(\tau \rightarrow \mu \gamma)$.

- The UT anomaly can be solved by means of negative NP effects in $\Delta M_{d} / \Delta M_{s}$ which, in turn, also indirectly enhance $\epsilon_{K}$ via the increased value of $R_{t}$. This scenario implies a lower bound for $\operatorname{BR}(\tau \rightarrow \mu \gamma)$ within the SuperB reach and large values for the angle $\gamma$ and it will be probed or falsified quite soon at the $\mathrm{LHCb}$.

- $\operatorname{Both} \operatorname{BR}\left(B_{s} \rightarrow \mu^{+} \mu^{-}\right)$and $\operatorname{BR}\left(B_{d} \rightarrow \mu^{+} \mu^{-}\right)$can reach large non-standard values. However, sizable departures from the $\mathrm{MFV}$ prediction $\mathrm{BR}\left(B_{s} \rightarrow\right.$ $\left.\mu^{+} \mu^{-}\right) / \operatorname{BR}\left(B_{d} \rightarrow \mu^{+} \mu^{-}\right) \approx\left|V_{t s} / V_{t d}\right|^{2}$ would imply large values for $\operatorname{BR}(\tau \rightarrow \mu \gamma)$, well within the SuperB reach.

- The dileptonic asymmetry $A_{\mathrm{SL}}^{b}$ can sizably depart from the SM expectations but the large value reported by the Tevatron [14] cannot be accounted for within the $S S U(5)_{R N}$ model. In particular, we find that $A_{\mathrm{SL}}^{b} \approx 0.5 A_{\mathrm{SL}}^{s}$ since $A_{\mathrm{SL}}^{d}$ remains SM-like.

- The asymmetry $S_{\phi K_{S}}$ can sizably depart from the SM expectations and it turns out to be correlated with $S_{\psi \phi}$. In particular, it is possible to simultaneously account for an enhancement of $S_{\psi \phi}$ and a suppression of $S_{\phi K_{S}}$ (relative to $S_{\psi K_{S}}$ ) as required by the data. This is in contrast to the SUSY flavour models discussed in Ref. 19. Moreover, the asymmetries $S_{\eta^{\prime} K_{S}}$ and $S_{\phi K_{S}}$ exhibit opposite deviations with respect to the SM expectations.

Finally, we provided a "DNA-Flavour Test" (proposed in Ref. [19]) for the $S S U(5)_{R N}$ model, with the aim of showing a tool to distinguish between NP scenarios, once additional data on flavour changing processes become available.

As shown in tab. II, further important predictions of the $S S U(5)_{R N}$ model are that i) $S_{\psi K_{S}}$ remains SM-like to a very good extent (therefore, the solution of the UT anomaly by means of $\mathrm{CPV}$ effects in $b \rightarrow d$ mixing is not possible), ii) CPV effects in $D^{0}-\bar{D}^{0}$ are negligibly small, and iii) $B R\left(K_{L}^{0} \rightarrow \pi^{0} \nu \bar{\nu}\right)$ and $B R\left(K^{+} \rightarrow \pi^{+} \nu \bar{\nu}\right)$ also remain SM-like.

In conclusion, the above results show the richness which is present in flavour physics once we embed a GUT group within a gravity mediated SUSY breaking scenario. It will be exciting to monitor upcoming results from the Tevatron, LHC(b), the MEG experiment at PSI and SuperB machines to establish whether some patterns of deviations from the SM expectations we have pointed out in this work are at work or not.

The interplay of all these efforts with the direct searches for NP will be most exciting.

Acknowledgments: AJB would like to thank the Particle Theory Institute of Vienna University for its hospitality during the final steps of this work. This work has been supported in part by the Cluster of Excellence "Origin and Structure of the Universe" and by the German Bundesministerium für Bildung und Forschung under contract $05 \mathrm{H} 09 \mathrm{WOE}$.
[1] H. Georgi and S. L. Glashow, Phys. Rev. Lett. 32 (1974) 438.

[2] S. Dimopoulos, S. Raby and F. Wilczek, Phys. Rev. D 24 (1981) 1681; S. Dimopoulos and H. Georgi, Nucl. Phys. B 193 (1981) 150; L. E. Ibanez and G. G. Ross, Phys.
Lett. B 105 (1981) 439.

[3] P. Minkowski, Phys. Lett. B67 (1977) 421; M. GellMann, P. Ramond, and R. Slansky in Supergravity, p. 315, edited by F. Nieuwenhuizen and D. Friedman, North Holland, Amsterdam, 1979; T. Yanagida, Proc. of the 
Workshop on Unified Theories and the Baryon Number of the Universe, edited by O. Sawada and A. Sugamoto, KEK, Japan 1979; R. N. Mohapatra, G. Senjanović, Phys. Rev. Lett. 44 (1980) 912.

[4] L. J. Hall, V. A. Kostelecky and S. Raby, Nucl. Phys. B 267, 415 (1986).

[5] F. Borzumati and A. Masiero, Phys. Rev. Lett. 57, 961 (1986).

[6] R. Barbieri and L. J. Hall, Phys. Lett. B 338 (1994) 212; R. Barbieri, L. J. Hall and A. Strumia, Nucl. Phys. B 445 (1995) 219.

[7] S. Baek, T. Goto, Y. Okada and K. i. Okumura, Phys. Rev. D 63, 051701 (2001); T. Moroi, JHEP 0003 (2000) 019; D. Chang, A. Masiero and H. Murayama, Phys. Rev. D 67, 075013 (2003); J. Hisano and Y. Shimizu, Phys. Lett. B 565 (2003) 183 arXiv:hep-ph/0303071; M. Ciuchini, A. Masiero, L. Silvestrini, S. K. Vempati and O. Vives, Phys. Rev. Lett. 92 (2004) 071801 arXiv:hepph/0307191]; S. Jager and U. Nierste, Eur. Phys. J. C 33 (2004) S256 arXiv:hep-ph/0312145; M. Ciuchini, A. Masiero, P. Paradisi, L. Silvestrini, S. K. Vempati and O. Vives, Nucl. Phys. B 783 (2007) 112 arXiv:hep$\mathrm{ph} / 0702144$.

[8] E. Lunghi and A. Soni, Phys. Lett. B 666, 162 (2008) arXiv:0803.4340 [hep-ph]]; E. Lunghi and A. Soni, arXiv:1010.6069 [hep-ph].

[9] A. J. Buras and D. Guadagnoli, Phys. Rev. D 78, 033005 (2008) arXiv:0805.3887 [hep-ph]].

[10] A. Lenz et al., arXiv:1008.1593 [hep-ph].

[11] A. Bevan et al. [UTfit Collaboration], arXiv:1010.5089 [hep-ph].

[12] T. Aaltonen et al. [CDF Collaboration], Phys. Rev. Lett. 100 (2008) 161802 arXiv:0712.2397 [hep-ex]].

[13] V. M. Abazov et al. [D0 Collaboration], Phys. Rev. Lett. 101 (2008) 241801 [arXiv:0802.2255 [hep-ex]].

[14] V. M. Abazov et al. [D0 Collaboration], arXiv:1005.2757.

[15] M. Passera, J. Phys. G 31 (2005) R75; Nucl. Phys. Proc. Suppl. 155 (2006) 365.

[16] M. Passera, W. J. Marciano and A. Sirlin, Phys. Rev. D 78, 013009 (2008).

[17] J. Hisano, D. Nomura and T. Yanagida, Phys. Lett. B 437 (1998) 351 arXiv:hep-ph/9711348; J. Hisano and D. Nomura, Phys. Rev. D 59 (1999) 116005 arXiv:hep$\mathrm{ph} / 9810479$.

[18] J. K. Parry and H. h. Zhang, Nucl. Phys. B 802 (2008) 63 arXiv:0710.5443 [hep-ph]]; T. Goto, Y. Okada, T. Shindou and M. Tanaka, Phys. Rev. D 77 (2008) 095010 arXiv:0711.2935 [hep-ph]]; J. Hisano and Y. Shimizu, Phys. Lett. B 669 (2008) 301 arXiv:0805.3327 [hep-ph]]; P. Ko, J. h. Park and M. Yamaguchi, JHEP 0811 (2008) 051 arXiv:0809.2784 [hep-ph]]; S. Trine, S. Westhoff and S. Wiesenfeldt, JHEP 0908 (2009) 002 arXiv:0904.0378 [hep-ph]]; B. Dutta, Y. Mimura and Y. Santoso, Phys. Rev. D 82 (2010) 055017 arXiv:1007.3696 [hep-ph]]; C. Biggio and L. Calibbi, JHEP 1010 (2010) 037 arXiv:1007.3750 [hep-ph]].

[19] W. Altmannshofer, A. J. Buras, S. Gori, P. Paradisi and D. M. Straub, Nucl. Phys. B 830 (2010) 17 arXiv:0909.1333 [hep-ph]].

[20] T. Goto, N. Kitazawa, Y. Okada and M. Tanaka, Phys. Rev. D 53 (1996) 6662 arXiv:hep-ph/9506311; N. G. Deshpande, B. Dutta and S. Oh, Phys. Rev. Lett. 77 (1996) 4499 arXiv:hep-ph/9608231; J. P. Silva and L. Wolfenstein, Phys. Rev. D 55 (1997) 5331 arXiv:hep- ph/9610208; A. G. Cohen, D. B. Kaplan, F. Lepeintre and A. E. Nelson, Phys. Rev. Lett. 78 (1997) 2300 arXiv:hep-ph/9610252; Y. Grossman, Y. Nir and M. P. Worah, Phys. Lett. B 407 (1997) 307 arXiv:hep$\mathrm{ph} / 9704287$.

[21] A. J. Buras, P. Gambino, M. Gorbahn, S. Jager and L. Silvestrini, Phys. Lett. B 500 (2001) 161 arXiv:hep$\mathrm{ph} / 0007085$.

[22] A. J. Buras, D. Guadagnoli and G. Isidori, Phys. Lett. B 688 (2010) 309 arXiv:1002.3612 [hep-ph]]

[23] J. Brod and M. Gorbahn, arXiv:1007.0684 [hep-ph].

[24] M. Bona et al. [UTfit Collaboration], JHEP 0603 (2006) 080 arXiv:hep-ph/0509219.

[25] J. Laiho, E. Lunghi and R. S. Van de Water, Phys. Rev. D 81 (2010) 034503 arXiv:0910.2928 [hep-ph]].

[26] D. J. Antonio et al. [RBC Collaboration and UKQCD Collaboration], Phys. Rev. Lett. 100 (2008) 032001 arXiv:hep-ph/0702042 . C. Aubin, J. Laiho and R. S. Van de Water, Phys. Rev. D 81 (2010) 014507 arXiv:0905.3947 [hep-lat]]. T. Bae et al., arXiv:1008.5179 [hep-lat]. M. Constantinou et al. [ETM Collaboration], arXiv:1009.5606 [hep-lat]. T. Bae et al., arXiv:1008.5179 [hep-lat].

[27] C. Amsler et al. [Particle Data Group], Phys. Lett. B 667 (2008) 1 .

[28] S. Herrlich and U. Nierste, Nucl. Phys. B 476 (1996) 27 arXiv:hep-ph/9604330.

[29] A. J. Buras, M. Jamin and P. H. Weisz, Nucl. Phys. B 347 (1990) 491.

[30] I. Allison et al. [HPQCD Collaboration], Phys. Rev. D 78 (2008) 054513 arXiv:0805.2999 [hep-lat]].

[31] CDF Collaboration and D0 Collaboration, arXiv:1007.3178 [hep-ex].

[32] M. Bona et al. [UTfit Collaboration], JHEP 0803 (2008) 049 arXiv:0707.0636 [hep-ph]]. Updates available on http://www.utfit.org.

[33] K. Nakamura et al. [Particle Data Group], J. Phys. G 37 (2010) 075021.

[34] S. Bethke, Eur. Phys. J. $\quad$ C $64 \quad$ (2009) 689 arXiv:0908.1135 [hep-ph]].

[35] E. Barberio et al. [Heavy Flavor Averaging Group], arXiv:0808.1297 [hep-ex].

[36] J. A. Casas and A. Ibarra, Nucl. Phys. B 618, 171 (2001) arXiv:hep-ph/0103065.

[37] J. Hisano, M. Nagai, P. Paradisi and Y. Shimizu, JHEP 0912 (2009) 030 arXiv:0904.2080 [hep-ph]].

[38] A. Rossi, Phys. Rev. D 66 (2002) 075003 arXiv:hep$\mathrm{ph} / 0207006$.

[39] For a recent detailed discussion, please see F. Borzumati and T. Yamashita, arXiv:0903.2793 [hep-ph].

[40] A. J. Buras, S. Jager and J. Urban, Nucl. Phys. B 605 (2001) 600 arXiv:hep-ph/0102316.

[41] A. Lenz and U. Nierste, JHEP. 0706, 072 (2007).

[42] Z. Ligeti, M. Papucci and G. Perez, Phys. Rev. Lett. 97 (2006) 101801 arXiv:hep-ph/0604112.

[43] Y. Grossman, Y. Nir and G. Perez, Phys. Rev. Lett. 103 (2009) 071602 arXiv:0904.0305 [hep-ph]].

[44] A. J. Buras, P. H. Chankowski, J. Rosiek and L. Slawianowska, Nucl. Phys. B 619 (2001) 434 arXiv:hepph/0107048; Phys. Lett. B 546 (2002) 96 arXiv:hepph/0207241; Nucl. Phys. B 659 (2003) 3 arXiv:hepph/0210145; M. Gorbahn, S. Jager, U. Nierste and S. Trine, arXiv:0901.2065 [hep-ph].

[45] G. Buchalla et al., JHEP 0509, 074 (2005) arXiv:hep- 
$\mathrm{ph} / 0503151$.

[46] J. Hisano, M. Nagai and P. Paradisi, Phys. Rev. D 80 (2009) 095014 arXiv:0812.4283 [hep-ph]]; J. Hisano, M. Nagai and P. Paradisi, Phys. Rev. D 78 (2008) 075019 arXiv:0712.1285 [hep-ph]]; J. Hisano, M. Nagai and P. Paradisi, Phys. Lett. B 642 (2006) 510 arXiv:hep$\mathrm{ph} / 0606322$.

[47] M. Pospelov and A. Ritz, Phys. Rev. Lett. 83 (1999) 2526; Nucl. Phys. B 573 (2000) 177; Phys. Rev. D 63 (2001) 073015.

[48] D. A. Demir, M. Pospelov and A. Ritz, Phys. Rev. D 67 (2003) 015007.

[49] D. A. Demir, O. Lebedev, K. A. Olive, M. Pospelov and A. Ritz, Nucl. Phys. B 680 (2004) 339.

[50] K. A. Olive, M. Pospelov, A. Ritz and Y. Santoso, Phys. Rev. D 72 (2005) 075001.

[51] G. Degrassi, E. Franco, S. Marchetti and L. Silvestrini, JHEP 0511 (2005) 044 arXiv:hep-ph/0510137.

[52] J. Hisano, T. Moroi, K. Tobe and M. Yamaguchi, Phys.
Rev. D 53 (1996) 2442 arXiv:hep-ph/9510309.

[53] J. Hisano and K. Tobe, Phys. Lett. B 510 (2001) 197 arXiv:hep-ph/0102315; G. Isidori, F. Mescia, P. Paradisi and D. Temes, Phys. Rev. D 75 (2007) 115019 arXiv:hep-ph/0703035.

[54] M. Misiak et al., Phys. Rev. Lett. 98, 022002 (2007); M. Misiak and M. Steinhauser, Nucl. Phys. B 764, 62 (2007).

[55] W. Altmannshofer, A. J. Buras and P. Paradisi, Phys. Lett. B 669 (2008) 239 arXiv:0808.0707 [hep-ph]].

[56] W. S. Hou, M. Nagashima and A. Soddu, Phys. Rev. D 72 (2005) 115007 arXiv:hep-ph/0508237.

[57] A. Soni, A. K. Alok, A. Giri, R. Mohanta and S. Nandi, Phys. Lett. B 683 (2010) 302 arXiv:0807.1971 [hep-ph]].

[58] A. J. Buras, B. Duling, T. Feldmann, T. Heidsieck, C. Promberger and S. Recksiegel, JHEP 1009 (2010) 106. arXiv:1002.2126 [hep-ph]]. 